\title{
A reticular chemistry guide for the design of periodic solids
}

\author{
Hao Jiang ${ }^{1}$, Dalal Alezi ${ }^{2}$ and Mohamed Eddaoudi ${ }^{1, \dagger}$ \\ ${ }^{1}$ Functional Materials Design, Discovery and Development Research Group (FMD ${ }^{3}$ ), \\ Advanced Membranes and Porous Materials Center (AMPMC), Division of Physical \\ Sciences and Engineering, King Abdullah University of Science and Technology (KAUST), \\ Thuwal, Kingdom of Saudi Arabia. \\ ${ }^{2}$ Department of Chemistry, Faculty of Science, King Abdulaziz University, Jeddah, \\ Kingdom of Saudi Arabia. \\ †e-mail: mohamed.eddaoudi@kaust.edu.sa
}

\section{Online summary}

The development of structural design methodologies in reticular chemistry promotes the discovery of periodic solids, such as metal-organic frameworks. In this Review, we highlight the well-developed and cutting-edge structural design methodologies, focusing on the role of building blocks, targeted nets and isoreticular chemistry.

\footnotetext{
Abstract | Reticular chemistry — the linking of well-defined molecular building blocks by strong bonds into crystalline extended frameworks - has enabled the synthesis of diverse metal-organic frameworks (MOFs) and covalent organic frameworks in which the pore shape, size and functionality can be tailored towards specific applications. Structural design methodologies are based on three main requisites — building blocks, targeted nets and isoreticular chemistry. In this Review, we highlight the well-developed and cutting-edge methodologies in reticular chemistry for the structural design and discovery of periodic solids. We illustrate the diversity of building blocks and delineate the suitable blueprint nets - namely, edge-transitive nets - for the design of MOFs. These edge-transitive nets are classified into three categories to help rationalize existing MOFs and to provide guidelines for the design of new structures. Two emerging topological concepts, namely the merged net approach and netcoded building units, are highlighted for their potential in synthesizing intricate or multicomponent MOFs. We also consider isoreticular design strategies for the modification,
} 
expansion and contraction of building blocks, and identify challenges and opportunities in the assembly of increasingly intricate frameworks.

\section{[H1] Introduction}

Although many periodic structures are discovered by serendipity, the rational molecular-level design and assembly of periodic materials is a long-term pursuit of material scientists. Over the past two decades, reticular chemistry has been widely used for the prediction and design of various periodically extended structures ${ }^{1-6}$. Reticular chemistry permits the geometry-guided pre-design of periodic frameworks, such as metal-organic frameworks (MOFs) and covalent organic frameworks (COFs), by connecting pre-selected building blocks.

There are three main requisites for reticular design: building blocks, targeted nets and isoreticular chemistry. In order to design and direct the construction of MOFs, it is essential to understand the structural features of their main constituents, namely building blocks. The diversity of building blocks provides myriad opportunities for the practice of reticular chemistry and MOF design. Building blocks can be categorized as molecular building blocks (MBBs) or supermolecular building blocks (SBBs). Readily accessible inorganic MBBs, such as single-metal-ion-based building blocks, metal clusters and infinite rods, are the primary requisite for the design of MOFs. Inorganic-organic zero-periodic SBBs or two-periodic supermolecular building layers (SBLs) provide an alternative for the design of more intricate MOFs and an approach to forming highly connected building blocks that are not readily accessible through the conventional MBB route. The discovery of new, readily accessible building blocks with a specific geometry is often the key to the design and discovery of new platforms.

Nets (or graphs) provide the blueprints of pre-selected platforms for materials design and construction in reticular chemistry. In this Review, we use the names of nets as employed in the Reticular Chemistry Structure Resource $(\mathrm{RCSR})^{7}$ database, a searchable database of plausible nets for the design of periodic materials. Although thousands of new MOF structures are reported every year, the majority of these are based on a limited number of nets. Evidently, not all nets are ideal design targets for reticular chemistry. A key property of a net is its transitivity [pqrs], which denotes that there are $p$ kinds of vertices (or nodes), $q$ kinds of edges, $r$ kinds of faces and $s$ kinds of tiles. Edge-transitive nets - nets with one type of edge (transitivity [11rs] or [21rs]) — are the most widely observed underlying nets of reported materials and are regarded as ideal targets for the design and construction of new structures in reticular chemistry ${ }^{8,9}$. Note that any periodic structure can be described by reducing its building 
blocks into nodes and spacers; the coordination of the simplified nodes defines the underlying net of the structure, and thus the structure is associated with a net with a particular underlying topology. Nets containing $m$-coordinated $(m-\mathrm{c})$ and $n$-coordinated $(n$-c) nodes are denoted as $(m, n)$-coordinated $((m, n)-c)$. For example, pcu is a 6-coordinated (6-c) net containing 6-c nodes, and rht is a $(3,24)$-c net containing 3-c nodes and 24-c nodes. An augmented net (net-a) is generated by substituting an $n$-c node in the original net with a group of vertices (polygon or polyhedron) corresponding to the coordination figure of the vertex ${ }^{7}$. For example, the $(3,5)-\mathrm{c}$ rht-a net is generated by substituting the 3-c nodes of rht with triangles (three new 3-c nodes) and the 24-c nodes with rhombicuboctahedra (24 5-c nodes).

Typically, MOFs based on edge-transitive nets contain only one type of inorganic MBB and one type of organic MBB, although multi-component materials containing mixed clusters or mixed linkers have been reported through partial substitution of the organic or inorganic building blocks. An approach to accessing multi-component materials by design has also been introduced based on merged nets - that is, nets that split into two or more nets with at least one net being three-periodic ${ }^{10}$. In this Review, we limit the discussion of multi-component materials to those based on merged nets, with emphasis on targeting more intricate structures.

Isoreticular design strategies are methods for the modification, replacement, expansion and contraction of building blocks, and for controlling net platforms to attain desired properties and applications. The multivariate (MTV) strategy can be applied to simultaneously introduce multiple functional groups by using building blocks with the same size and connectivity metrics but with distinct functional groups or metal ions. The expansion and contraction of merged-net materials is limited by a size constraint - the ratio constant of the edges in a merged net that has to be considered during the design of intricate structures.

This Review is organized into three main sections. We first provide an overview of building blocks, highlighting some of the key geometries awaiting discovery. Second, we delineate the fundamental design targets - edge-transitive nets - and provide representative structures to demonstrate the role of underlying nets in the construction of materials from building blocks. We also discuss the assembly of multi-component materials based on merged nets and highlight important examples of non-edge-transitive platforms. Third, we outline isoreticular design strategies, including the MTV approach and methodologies for building-block expansion and contraction. Finally, we provide our perspective on advancing reticular chemistry design strategies for the assembly of more intricate structures. 


\section{[H1] Building blocks}

\section{[H2] Single-metal-ion-based building blocks}

Single metal ions are an essential source of inorganic tetrahedral, square and octahedral building blocks, and are well known for their ability to construct zeolite-like MOFs (ZMOFs) ${ }^{11}$. Zeolitic imidazolate frameworks (ZIFs) with variable topologies can be synthesized by tuning the functional groups on imidazole ${ }^{12}$. More recently, it was found that the underlying topology and cage size of ZIFs can be tuned by controlling the shape and size of the functional group on the imidazolate linker, linker combinations and linker ratios ${ }^{13}$. Another approach to constructing ZMOFs involves linkers containing both N-donors and O-donors to form heterochelating moieties ${ }^{14,15}$. Like the replacement of $\mathrm{Si}^{4+}$ centres by $\mathrm{Al}^{3+}$ and $\mathrm{P}^{5+}$ in silicalites, the $\mathrm{Zn}^{2+}$ nodes in ZIFs can be replaced by $\mathrm{B}^{3+}$ together with either $\mathrm{Li}^{+}$or $\mathrm{Cu}^{+}$ions ${ }^{16,17}$, with the organic B-imidazolate complexes synthesized prior to MOF assembly.

A sizeable MOF family based on 6-c octahedral single metal ions has demonstrated excellent performance for $\mathrm{CO}_{2}$ capture and gas separation ${ }^{18-21}$. The representative examples are the SIFSIX MOFs: a series of materials based on square grid layers, constructed by connecting $\mathrm{M}^{2+}$ cations (such as $\mathrm{Cu}^{2+}, \mathrm{Zn}^{2+}, \mathrm{Ni}^{2+}$ ) and ditopic organic linkers, pillared with inorganic $\left[\mathrm{SiF}_{6}\right]^{2-}$ anions. The resulting $3 \mathrm{D}$ structures are based on the pcu net ${ }^{22}$ and contain 1D square channels amenable to pore-size tuning by changing the cations. For example, the pore-aperture size of the square channels in the SIFSIX-3-M series is $3.78 \AA$, $3.69 \AA$ and $3.48 \AA$ for SIFSIX3-Zn, SIFSIX-3-Ni and SIFSIX-3-Cu, respectively ${ }^{21,23,24}$. The affinity of these materials for $\mathrm{CO}_{2}$ adsorption increases on contraction of the pore size: SIFSIX-3-Cu exhibits the steepest $\mathrm{CO}_{2}$ adsorption isotherm at low concentrations and enhanced adsorption energetics. Structural analysis reveals that the contraction of the channel decreases the distance between the pendant fluorine moieties within the channel, increasing the strength of $\mathrm{CO}_{2}$-framework interactions at lower concentrations ${ }^{24}$.

Replacing the $\left[\mathrm{SiF}_{6}\right]^{2-}$ pillars in SIFSIX-3-Ni with larger and more nucleophilic inorganic $\left[\mathrm{NbOF}_{5}\right]^{2-}$ pillars yields a more robust and highly water stable analogue called NbOFFIVE-1$\mathrm{Ni}^{25-27}$. Compared with its $\left[\mathrm{SiF}_{6}\right]^{2-}$ analogue, NbOFFIVE-1-Ni exhibits shorter distances between the adjacent pendant fluorine moieties within the channels, resulting in contraction of the pore-aperture size to $3.21 \AA$. NbOFFIVE-1-Ni has the highest $\mathrm{CO}_{2}$ gravimetric and volumetric uptake among physical adsorbents at $400 \mathrm{ppm}$ of $\mathrm{CO}_{2}$ and $298 \mathrm{~K}$, with moderate energy requirements for regeneration.

\section{[H2] Metal-cluster building blocks}


The connectivity of single metal ions is generally low $(<8)$ with only a limited number of geometries. By contrast, metal clusters afford diverse shapes of building blocks (Fig. 1), especially highly connected building blocks. The ability to reliably obtain a required metalcluster MBB is central to the structural design of MOFs. The points of extension of a MBB define the geometry and connectivity of the resultant secondary building unit (SBU). Note that we refer to an MBB as a chemical moiety with an associated SBU that codes the geometrical information expressed by the points of extension of the MBB. Building units with connectivity $n$ are denoted as $n$-connected $(n-\mathrm{c})$, where $n \geq 2$.

Depending on the connectivity of the building block, some MBBs can form SBUs with different geometries and connectivity. MBBs that contain open metal sites can associate with extra linkers, producing building units with higher connectivity. By contrast, some MBBs can yield SBUs with lower connectivity when some points of extension are capped by a terminal ligand. In this section, we describe representative metal-cluster MBBs, focusing on those that can be most reliably obtained.

One of the most widely deployed MBBs, the binuclear metal paddlewheel $\mathrm{M}_{2} \mathrm{~L}_{4}$ (where $\mathrm{M}$ $=\mathrm{Cu}^{2+}, \mathrm{Zn}^{2+}$, among others, and $\mathrm{L}$ is a linker), typically represents a 4-c square SBU (Fig. 1a). HKUST-1, based on a $(3,4)-c$ tbo net is constructed by linking a 4-c $\mathrm{Cu}$ paddlewheel and the 3 -c tritopic linker benzene-1,3,5-tricarboxylate $(\mathrm{BTC})^{28}$. The open metal sites provide extra points of extension for this cluster, which can connect to two other linkers ( $\mathrm{L}^{\prime}$ ) perpendicular to the square plane. The resulting cluster, $\mathrm{M}_{2} \mathrm{~L}_{4} \mathrm{~L}_{2}^{\prime}$, provides a 6-c octahedral building unit (Fig. 1a), and is useful for building pillared MOFs in which the paddlewheels form two-periodic layers that are linked through pyridine moieites ${ }^{29}$. Linking only one pyridine moiety to the paddlewheel forms a $\mathrm{M}_{2} \mathrm{~L}_{4} \mathrm{~L}^{\prime}$ cluster that represents a 5-c pyramidal building unit, which can be used to construct SBB-based MOFs ${ }^{30}$.

The trinuclear clusters $\mathrm{M}_{3} \mathrm{~L}_{3}\left(\mathrm{M}=\mathrm{Cu}^{2+}, \mathrm{Ag}^{+}\right.$, among others $)$often represent 3-c trigonal building units ${ }^{31}$ (Fig. 1b). For example, rht-MOF-1 is formed from tetrazole-functionalized isophthalate as the organic linker, a 24-c building unit based on a $\mathrm{Cu}$ paddlewheel and isophthalates and trinuclear clusters based on $\mathrm{Cu}^{2+}$ and tetrazolates ${ }^{32}$. When each open metal site connects to another linker, the resulting trinuclear cluster $\mathrm{M}_{3} \mathrm{~L}_{3} \mathrm{~L}_{3}^{\prime}$ (Fig. 1b) represents a 6c hexagonal building unit. This 6-c building unit combines with a pyrazole-carboxylate linker to form hxg-MOF ${ }^{33}$.

The trinuclear clusters $\mathrm{M}_{3} \mathrm{~L}_{6}\left(\mathrm{M}=\mathrm{Al}^{3+}, \mathrm{Mg}^{2+}, \mathrm{Sc}^{3+}, \mathrm{Cr}^{2+}, \mathrm{Cr}^{3+}, \mathrm{Mn}^{2+}, \mathrm{Mn}^{3+}, \mathrm{Fe}^{2+}, \mathrm{Fe}^{3+}\right.$, $\mathrm{Co}^{2+}, \mathrm{Ni}^{2+}, \mathrm{Ga}^{3+}, \mathrm{In}^{3+}$, among others) typically represent 6 -c trigonal prismatic building units ${ }^{34-}$ 
38 (Fig. 1c), which are the fundamental building units for the construction of edge-transitive nets based on 6-c nodes, such as $\mathbf{a c s}^{36}, \mathbf{s o c}^{34,35}, \mathbf{s t p}^{39}$ and nia ${ }^{40}$. Note that building units with the same connectivity but different geometries can interconvert. For example, soc-MOF, based on 4-c square and 6-c octahedral building units, can afford the points of extension to be a 4-c rectangular and 6-c trigonal prismatic building unit ${ }^{34,41}$. Through the open metal sites, the $\mathrm{M}_{3} \mathrm{~L}_{6}$ cluster can connect to an additional three linkers. The resulting cluster, $\mathrm{M}_{3} \mathrm{~L}_{6} \mathrm{~L}_{3}^{\prime}$, is a 9-c tricapped trigonal prism (also known as Johnson solid $J_{51}$ ) (Fig. 1c). These 9-c building blocks have been used to construct mixed-linker MOFs (discussed further below) based on merged nets $^{42-45,46}$.

The tetranuclear clusters $\mathrm{M}_{4} \mathrm{~L}_{6}\left(\mathrm{M}=\mathrm{Zn}^{2+}, \mathrm{Co}^{2+}, \mathrm{Y}^{3+}, \mathrm{Ln}^{3+}\right.$, among others $)$ represent 6-c octahedral building units (Fig. 1d), and were the first MBBs to be systematically explored for the construction of MOF-5 and its isoreticular series, IRMOFs ${ }^{47,48}$. The organic linkers can be O-donor carboxylates ${ }^{47,48}$ or $\mathrm{N}$-donors ${ }^{49}$. Moreover, one $\mathrm{Zn}$ centre in the tetranuclear $\mathrm{Zn}$ cluster $\left(\mathrm{Zn}_{4}\left(\mu_{4}-\mathrm{O}\right)\left(\mathrm{O}_{2} \mathrm{C}-\right)_{6}\right)$ can be substituted by another metal ion ${ }^{50}$, such as $\mathrm{Ti}^{3+}, \mathrm{V}^{2+}, \mathrm{V}^{3+}, \mathrm{Cr}^{2+}, \mathrm{Cr}^{3+}$, $\mathrm{Mn}^{2+}$ or $\mathrm{Fe}^{2+}$, affording $\mathrm{MZn}_{3}\left(\mu_{4}-\mathrm{O}\right)\left(\mathrm{O}_{2} \mathrm{C}^{-}\right)_{6}$ clusters. The $\mathrm{Zn}$ atoms in the tetranuclear $\mathrm{Zn}$ cluster are typically bridged by a $\mu_{4}-\mathrm{O}$ group ${ }^{47-49}$, whereas the metal atoms in rare-earth (RE) clusters are typically linked by four $\mu_{3}-\mathrm{OH}$ moieties, giving $\left[(\mathrm{RE})_{4}\left(\mu_{3}-\mathrm{OH}\right)_{4}\left(\mathrm{O}_{2} \mathrm{C}-\right)_{6}\right]^{2+}$ clusters $^{51}$.

The planar tetranuclear clusters $\mathrm{M}_{4} \mathrm{~L}_{8}\left(\mathrm{M}=\mathrm{Mn}^{2+}, \mathrm{Co}^{2+}, \mathrm{Cu}^{2+}, \mathrm{Cd}^{2+}, \mathrm{Ni}^{2+}, \mathrm{Y}^{3+}, \mathrm{Ln}^{3+}\right.$, among others) represent 8-c cuboid building units (Fig. 1e). $\mathrm{M}_{4} \mathrm{~L}_{8}$ building blocks are combined with ditopic linkers to construct $8-\mathbf{c}$ bcu-MOFs ${ }^{52}$ and reo-MOFs ${ }^{53}$, and tritopic linkers to form $(3,8)-$ c the-MOFs ${ }^{54}$. A mixed-metal the-MOF, MUV-10, based on the $\mathrm{Ti}_{2} \mathrm{Ca}_{2}\left(\mu_{3}-\mathrm{O}\right)_{2}\left(\mathrm{H}_{2} \mathrm{O}\right)_{4}\left(\mathrm{O}_{2} \mathrm{C}-\right)_{8}$ cluster, has also been reported ${ }^{55}$.

The hexanuclear clusters $\mathrm{M}_{6} \mathrm{~L}_{12}\left(\mathrm{M}=\mathrm{Y}^{3+}, \mathrm{Ln}^{3+}, \mathrm{Zr}^{4+}, \mathrm{Ce}^{4+}\right.$, among others $)$ typically represent 12-c cuboctahedral building units ${ }^{56-59}$ (Fig. 1f). The first $\mathrm{Zr}_{6}$-based MOF, UiO-66, is one of the most widely explored MOFs owing to its excellent stability and simple synthesis conditions $^{57}$. The subsequent discovery of hexanuclear rare-earth clusters paved the way to highly connected rare-earth MOFs ${ }^{58}$. Owing to the complicated coordination modes of rareearth ions, it is not straightforward to form highly symmetric rare-earth clusters. However, by functionalizing the 2-position of the benzoic acid moiety in the linker with a fluoro group, a series of rare-earth fcu-MOFs was obtained with hexanuclear rare-earth clusters instead of the infinite chains often observed in rare-earth $\mathrm{MOFs}^{58}$. Hexanuclear MBBs with sites that are capped with terminal ligands can also afford other polyhedrons, such as a 10-c elongated 
octahedron (Johnson solid $\left.J_{15}\right)^{60}, 8$-c cuboid ${ }^{60-62}, 6$-c hexagon ${ }^{63}, 6$-c trigonal antiprism ${ }^{10,60,64,65}$ (Fig. 1f) and 4-c rectangle $66,67$.

The octanuclear clusters $\mathrm{M}_{8} \mathrm{~L}_{12}\left(\mathrm{M}=\mathrm{Co}^{2+}, \mathrm{Ni}^{2+}, \mathrm{Zr}^{4+}\right.$, among others $)$ represent 12-c cuboctahedral building units ${ }^{68-70}$ (Fig. 1g). Ni-fcu-MOFs are formed from octanuclear Ni clusters and ditopic pyrazole-based linkers ${ }^{68}$. This type of fcu-MOF has also been obtained with octanuclear Co clusters ${ }^{69}$. Octanuclear $\mathrm{Zr}$ clusters are rarer than hexanuclear $\mathrm{Zr}$ clusters; one example is PCN-221, a ftw-MOF that comprises octanuclear $\mathrm{Zr}$ clusters and porphyrin tetracarboxylate linkers ${ }^{70}$. Note that although both $\mathrm{M}_{6} \mathrm{~L}_{12}$ and $\mathrm{M}_{8} \mathrm{~L}_{12} \mathrm{MBBs}$ represent 12-c cuboctahedral building units, the required torsion angle of the linking group in the organic linkers is different. ftw-MOFs based on hexanuclear clusters require tetratopic linkers with $0^{\circ}$ torsion $^{71}$, whereas those based on octanuclear clusters require tetratopic linkers with a torsion angle of $\sim 90^{\circ}\left(\right.$ ref. $\left.^{70}\right)$.

The planar octanuclear clusters $\mathrm{M}_{8} \mathrm{~L}_{16}\left(\mathrm{M}=\mathrm{Al}^{3+}, \mathrm{Cr}^{3+}, \mathrm{Ti}^{4+}\right.$, among others) represent 16-c snub square antiprismatic (Johnson solid $J_{85}$ ) building units with each metal node coordinated to four carboxylates ${ }^{72}$ (Fig. 1h). One example of a MOF with this 16-c building unit is the mixed-linker MOF based on the (3,16)-c skl net, MOF-520-BPDC, which contains the tritopic linker 4,4',4'-benzene-1,3,5-triyl-tribenzoate (BTB) and the ditopic linker biphenyl-4,4'dicarboxylate (BPDC) ${ }^{72}$. MOF-520-BPDC was synthesized using a two-step reaction: MOF520 with only the BTB linker, and based on the $(3,12)$-c fon net with unsaturated clusters, was reacted with BPDC to increase the connectivity of the clusters from 12-c to $16-\mathrm{c}^{73,74}$. The planar octanuclear clusters with only 12 linkers connected $\left(\mathrm{M}_{8} \mathrm{~L}_{12}\right)$ (Fig. 1h) can also represent 12-c distorted cuboctahedrons ${ }^{75,76}$. One early example is CAU-1 $\left(\right.$ ref. $^{75}$ ), an Al-MOF based on the 12-c fcu net with the amino-benzenedicarboxylate $\left(\mathrm{NH}_{2}\right.$-BDC) linker. $\mathrm{CAU}-1$ was isolated from the more common MIL-53- $\mathrm{NH}_{2}$ and MIL-101- $\mathrm{NH}_{2}$ phases by carefully tuning the metalto-linker ratio, solvents and aluminium salts. By using Ti as the metal centre and BDC as the linker, MIL-125 was obtained as the first Ti-MOF based on the 12-c fcu net ${ }^{76}$.

The nonanuclear clusters $\mathrm{M}_{9} \mathrm{~L}_{12}\left(\mathrm{M}=\mathrm{Y}^{3+}, \mathrm{Ln}^{3+}\right)$ often represent 12-c hexagonal prismatic building units ${ }^{62}$ (Fig. 1i), which were used for the construction of highly coordinated $(3,8,12)$ c pek-MOFs and $(3,12,12)-c$ aea-MOFs ${ }^{62}$. Nonanuclear clusters contain six extra connecting sites and, thus, can connect to other linkers, forming an 18-c elongated trigonal orthobicupola (Johnson solid $J_{35}$ ) $\mathrm{M}_{9} \mathrm{~L}_{12} \mathrm{~L}_{6}^{\prime}$ building unit (Fig. 1i). One representative example is gea-MOF1 (ref. $^{77}$ ), which is constructed from 18-c nonanuclear rare-earth clusters and 3-c trigonal tricarboxylate ligands. 


\section{[H2] Infinite rods and layers}

The design of MOFs based on one-periodic rods is more intricate than those based on zeroperiodic metal ions and clusters. Zero-periodic MBBs yield building units with a defined number of points of extension and geometry, whereas one-periodic rods offer building units with an infinite number of points of extension. The structural and topological analysis of rod MOFs is well established ${ }^{78}$. However, the design of MOFs from rod building blocks is less reliable than from polyhedral MBBs, partly because of the difficulty in predicting targeted nets based on rod building blocks. Moreover, access to rod building blocks is highly dependent on explicit organic linkers and synthesis conditions. Nevertheless, some rod building blocks constructed from multi-functional groups can be reliably obtained. For example, the MOF-74 family can be obtained from 6-c metal clusters and ditopic carboxylate linkers with an ortho hydroxy group ${ }^{79}$. By keeping the same rod building block but tuning the position of the hydroxy group on a 4,6-dihydroxy-1,3-benzene dicarboxylate ( $m$-DOBDC) linker, a structure with the formula $\mathrm{M}_{2}$ (m-DOBDC) was reported ${ }^{80}$. The linker can also be elongated to $4,4^{\prime}$-dihydroxy3,3'-biphenyl dicarboxylate ${ }^{81}$. Another example, STA-12, with the formula $\mathrm{Ni}_{2} \mathrm{~L}$, comprises an $N, N^{\prime}$-piperazine bismethylene phosphonate linker that contains both $\mathrm{O}-$ and $\mathrm{N}$ - functional groups $^{82,83}$. Isoreticular extension of this platform resulted in STA-16, with the formula $\mathrm{Co}_{2} \mathrm{~L}$, featuring the longer linker $N, N^{\prime}-4,4^{\prime}$-bipiperidine bismethylene phosphonate ${ }^{84}$.

The assembly of rod MOFs based on MOF-910, constructed from a unique heterotritopic organic linker with three distinct functional groups (phenylyne-1-benzoate, 3benzosemiquinonate and 5-oxidopyridine), has also been explored ${ }^{85}$. Two principles were derived from this study. First, polyheterotopic linkers are effective for selectively obtaining helical targets instead of straight rod building units. Second, the helical building units can be tuned by adjusting of the polyheterotopic linker.

In most cases, rod MOFs prevent structural interpenetration, which can result in welldefined 1D channels suitable for applications in selective separation. For example, a rod MOF with the formula $\mathrm{Fe}_{2}(\mathrm{BDP})_{3}\left(\mathrm{BDP}^{2-}=1\right.$,4-benzene dipyrazolate) separates hexane isomers according to the degree of branching ${ }^{86}$. Another rod MOF, NOTT-300, selectively binds $\mathrm{CO}_{2}$ and $\mathrm{SO}_{2}$ through exposed hydroxy groups within the pore system ${ }^{87}$. In contrast to one-periodic infinite rods, two-periodic infinite layers have been less explored for the construction of MOFs. Recently, two-periodic $\mathrm{MoS}_{2}$ layers were pillared with polytopic amine-based ligands to form three-periodic $\mathrm{MOFs}^{88}$. 


\section{[H2] Organic building blocks}

Organic building blocks, or linkers, are central to the design of MOFs, COFs and organic cages. The tunable geometries of organic building blocks provide a vast space for exploration. Linkers of certain geometries are urgently needed, especially those with a high connectivity.

Eight fundamental operations describe the changes in position of the end groups in organic building blocks: the identity operation, four in-plane operations (expansion, contraction, inplane rotation and in-plane translation) and three out-of-plane operations (torsion, out-of-plane rotation and out-of-plane translation) (Fig. 2a).

The identity, expansion and contraction operations of organic linkers are valuable for the practice of isoreticular chemistry. When a functional group is added to an organic linker without tuning the position or angle, the geometry of the linker remains the same and the change can be considered as an identity operation. When the size of the organic linker is changed, the whole structure will either expand or contract (discussed further below).

The two rotation operations produce bent linkers, which are essential for the construction of some special zero-periodic cages ${ }^{89-91}$, two-periodic layers ${ }^{92}$ and three-periodic frameworks ${ }^{93-}$ 95. Unlike in-plane rotation, out-of-plane rotation results in a dihedral angle between two planes of linking moieties ${ }^{96}$ (Fig. 2b). Some branched linkers also exhibit in-plane rotation of the linking group ${ }^{34,35,41,97}$.

Torsion of the linking group results in a twisted linker. For example MOF-101, an nboMOF, is constructed from a $\mathrm{Cu}$ paddlewheel and a twisted $o$-Br-BDC linker ${ }^{98}$. Furthermore, NU-1301, an intricate mesoporous uranium MOF, is constructed from simple twisted tritopic linkers ${ }^{99}$. The twisted ligand prevents the formation of edge-transitive srs or hcb structures, and forms a complicated nun structure comprising 816 uranium nodes and 816 organic linkers in one $173.3 \AA$ cubic unit cell — the largest unit cell of any non-biological material. In a recent examination of geometry mismatch in MOFs, the rotation and torsion angles of organic linkers were systematically measured, providing the most comprehensive database of the angletopology relationship to date ${ }^{100}$.

The in-plane translation of ditopic linkers result in zigzag linkers ${ }^{101,102}$. A systematic study of zigzag ditopic linkers afforded isoreticular Zr-MOFs based on the bcu net rather than the common fcu net ${ }^{101}$. Moreover, MOF-909, based on the $(3,3,6)$-c nht net, is a mixed-linker MOF with the tritopic BTB linker and the ditopic zigzag 3,3'-azoBDC linker. Metallocene- 
based ligands are examples of out-of-plane translation linkers (Fig. 2b), but these are less explored than in-plane translation linkers ${ }^{103}$.

\section{[H2] Supermolecular building blocks}

The SBB approach uses zero-periodic metal-organic polyhedrons (MOPs) as building blocks to construct MOFs with highly connected topologies. This approach was first introduced for the construction of rht-MOFs based on the $(3,24)$-c net as a means to access the 24-c building unit, which is difficult to obtain as a conventional inorganic $\mathrm{MBB}^{32}$. This section provides an overview of some SBBs that can be reliably obtained.

The $\left(\mathrm{M}_{2}\right)_{6} \mathrm{~L}_{12} \mathrm{~L}_{6}^{\prime} \mathrm{SBB}\left(\mathrm{M}=\mathrm{Cu}^{2+}, \mathrm{Mo}^{2+}, \mathrm{Ni}^{2+}, \mathrm{Co}^{2+}, \mathrm{Cr}^{2+}\right.$, among others $)$ can be regarded as an 18-c building unit: 12-c from the linkers and 6-c from the open metal sites. Octahedral $\left(\mathrm{M}_{2}\right)_{6} \mathrm{~L}_{12}$ was first constructed from a $\mathrm{Cu}_{2}$ paddlewheel and 2,2':5',2"'-terthiophene-5,5'dicarboxylate ${ }^{104}$. The construction of $\left(\mathrm{M}_{2}\right)_{6} \mathrm{~L}_{12}$-based MOPs was further explored by bridging various metal paddlewheel clusters, namely $\mathrm{Cu}$ (ref. ${ }^{105}$ ), Mo (ref. ${ }^{106}$ ), Co (ref. ${ }^{107}$ ) and $\mathrm{Cr}$ (ref. ${ }^{108}$ ), with the $9 H$-carbazole-3,6-dicarboxylate (CDC) linker (Fig. 1j). The first reported structure based on a $\left(\mathrm{M}_{2}\right)_{6} \mathrm{~L}_{12} \mathrm{SBB}$ was Ni-fcu-MOF, in which the SBB comprises six Ni dimers and twelve isophthalates ${ }^{109}$ (Fig. 3). A pcu-MOF based on the underlying pcu-a (cab) net was constructed by connecting a CDC-based SBB with a 4,4'-bipyridine linker ${ }^{105}$. The CDC-based SBB can also be used as a 12-c cuboctahedral building block by deploying the $\mathrm{N}$ atom in the carbazole ligands as a point of extension, as exemplified in the fcu-MOF, DUT-49, which can also be described as an nbo-MOF based on the nbo-derived $\mathbf{t f b}$ net $^{110}$ (Fig. 3). Using a flexible bicarbazole ligand (9,9'-([1,1'-biphenyl]-4,4'-diyl)bis( $9 H$-carbazole-3,6dicarboxylate) as the organic linker, DUT-49 exhibits rare negative gas-adsorption behaviour $^{111}$. The CDC-based SBB can also form ftw-MOFs by combining with tritopic ligands 9-(4-carboxyphenyl)-9H-carbazole-3,6-dicarboxylic acid (DUT-75) or 9-(4'-carboxy$\left[1,1^{\prime}\right.$-biphenyl]-4-yl)-9H-carbazole-3,6-dicarboxylic acid (DUT-76) ${ }^{112}$. DUT-76 shows a high pore volume of $3.25 \mathrm{~cm}^{3} \mathrm{~g}^{-1}$ and a high Brunauer-Emmett-Teller surface area of $6,344 \mathrm{~m}^{2} \mathrm{~g}^{-1}$.

The $\left(\mathrm{M}_{2}\right)_{12} \mathrm{~L}_{24} \mathrm{~L}_{12}^{\prime}$ SBBs $\left(\mathrm{M}=\mathrm{Cu}^{2+}, \mathrm{Zn}^{2+}, \mathrm{Mo}^{2+}, \mathrm{Ni}^{2+}, \mathrm{Co}^{2+}, \mathrm{Cr}^{2+}\right.$, among others $)$ can be used as 36-c building units: $24-\mathrm{c}$ from the linkers and 12-c from the open metal sites. MOP-1 was first obtained with a formula $\mathrm{Cu}_{24}(1,3-\mathrm{BDC})_{24}$ (solvent) (ref. $^{89}$ ). As an SBB, MOP-1 is typically shown as a 24-c rhombicuboctahedron ${ }^{32,113}$ (Fig. 1k). With only L' linked, the $\left(\mathrm{M}_{2}\right)_{12} \mathrm{~L}_{12}^{\prime} \mathrm{SBB}$ represents a 12-c cuboctahedral building unit. For example, a Zn-fcu-MOF is formed by linking Zn-based MOP-1 with the ditopic linker 1,4-diazabicyclo-[2.2.2]octane at 
the open metal $\operatorname{sites}^{30}$ (Fig. 3). When both the linker and open metal sites are linked, the saturated building block is regarded as a 36-c SBB, which can be used to construct the highly connected (3,36)-c txt-MOFs, based on the underlying txt-derived (3,5)-c pzh net ${ }^{114,115}$.

The $\left(\mathrm{Cu}_{2}\right)_{6} \mathrm{~L}_{12} \mathrm{~L}_{6}^{\prime}$ elongated trigonal orthobicupola (Johnson solid $J_{35}$ ) MOP was reported with mixed $120^{\circ}$ and $90^{\circ}$ linkers ${ }^{116}$. An intricate MOF, gea-MOF-2, is an example of a structure designed using the SBB approach ${ }^{92}$. By careful examination of the geometry of this MOP, a trefoil-like hexacarboxylate ligand with one $90^{\circ}$ arm and two $120^{\circ}$ arms was designed for the synthesis of $(3,18)$-c gea MOF, which can also be considered as a pillared two-periodic edgetransitive hxl net.

The exploration of new types of cages is crucial for the construction of highly connected MOFs. There are several potential SBBs that are still to be explored for the construction of MOFs. Namely, Goldberg polyhedral cages based on the assembly of $30 \mathrm{Pd}$ ions and 60 ligands ${ }^{90}$. Additionally, the emergence of various pure organic cages also expands the library of SBBs for the future practice of the SBB approach. For example, chiral covalent cages were used to obtain one-periodic nanotubes and three-periodic frameworks ${ }^{117}$.

\section{[H2] Supermolecular building layers}

The SBL approach uses two-periodic MOFs as building layers for the construction of threeperiodic $\mathrm{MOFs}^{92}$, and was introduced as a strategy for the design of pillared functional MOFs $^{29,118}$. The key to effectively deploying the SBL approach is to target readily accessible two-periodic edge-transitive nets, namely sql, kgm, hcb, kgd and hxl, as SBLs and to then crosslink the layers through accessible bridging sites using appropriate organic ligands to construct three-periodic porous MOFs ${ }^{29,92,118}$.

Three pillaring strategies have been explored to construct a plethora of pillared MOF structures: axial-to-axial (A-A), axial-to-ligand (A-L) and ligand-to-ligand (L-L) ${ }^{29,119,120}$. The A-A pillaring strategy exploits neighbouring accessible metal sites by replacing the terminal ligands, such as water or pyridine, with bridging ditopic ligands, resulting in crosslinking of the two-periodic MOF layers. The most well-studied examples of this strategy involve pillaring of MOFs with sql or kgm topology, which are mainly based on 4-c $\mathrm{M}_{2}\left(\mathrm{O}_{2} \mathrm{C}-\right)_{4}(\mathrm{~A})_{2}\left(\mathrm{M}=\mathrm{Cu}^{2+}\right.$, $\mathrm{Ni}^{2+}, \mathrm{Fe}^{2+}, \mathrm{Zn}^{2+}, \mathrm{Co}^{2+}$, among others; $\mathrm{A}=$ axial linker) paddlewheel $\mathrm{MBBs}$, from the axial site on the paddlewheel MBBs using linear ditopic or bifunctional ligands ${ }^{92,121}$. The $\mathrm{L}-\mathrm{L}$ pillaring strategy was introduced following topological analysis of the three-periodic tbo-MOF, HKUST-1 (ref. ${ }^{28}$ ), pinpointing an alternative approach to understanding and deconstructing the 
HKUST-1 structure into sql layers crosslinked by 4-c (quadrangular) inorganic MBB pillars ${ }^{118}$. The substitution of the inorganic MBBs by organic octatopic ligands (quadrangular pillars) enables the construction of non-interpenetrated isoreticular and functionalized analogues of HKUST-1, such as tbo-MOFs ${ }^{118,122,123}$. The L-A pillaring strategy requires the use of linear heterofunctional trigonal ligands containing the isophthalic moiety to form the targeted sql or kgm MOF layers, as well as a N-donor group to connect to neighbouring layers through coordination to open metal sites ${ }^{29,124,125}$.

The SBL pillaring approach based on highly connected $\mathrm{Zr}$ and rare-earth clusters is less explored (Fig. 4). We exemplify this approach by describing the doubly crosslinked kgm layer that is associated with the underlying pek net ${ }^{62}$. pek-MOF is a $(3,8,12)$-c net based on the assembly of two highly connected rare-earth clusters, namely nonanuclear $[(\mathrm{RE}))_{9}\left(\mu_{3}-\mathrm{OH}\right)_{12}\left(\mu_{3^{-}}\right.$ $\left.\mathrm{O})_{2}\left(\mathrm{O}_{2} \mathrm{C}-\right)_{12}\right]$ and hexanuclear $\left[(\mathrm{RE})_{6}(\mathrm{OH})_{8}\left(\mathrm{O}_{2} \mathrm{C}-\right)_{8}\right]$ serving as 12-c hexagonal prismatic and 8-c cuboid MBBs, respectively. The hexanuclear clusters are arranged in two-periodic kgm layers (Fig. 4a) with a connectivity twice that of the well-known $\mathrm{Cu}$ isophthalate $\mathbf{k g m}$ layers found in many MOFs ${ }^{92,97}$. The kgm layers are then intercalated by the 12-c hexagonal prismatic MBB to form pek-MOF-1. The hexanuclear clusters can also form distorted sql-SBLs with a twisted $120^{\circ}$ linker (Fig. 4a) and have been used to form a Zr-scu-MOF, PCN-606, with the L-L pillaring strategy ${ }^{61}$. A kgd-SBL (Fig. 4a) was reported with the hexanuclear Zr cluster and tritopic linker BTB ${ }^{126}$. These $2 \mathrm{D}$ networks catenate to form a 3D porous framework, which is the first Zr-MOF with 2D to 3D catenation. The nonanuclear cluster can form hxl-SBLs (Fig. $4 \mathrm{~b})$ with a $120^{\circ}$ linker, and were used for the construction of RE-gea-MOF-1 (ref. ${ }^{77}$ ). The nonanuclear cluster can also form hcb-SBLs (Fig. 4b) with a carbazole-based linker, as reported in the construction of RE-aea-MOF-1 (ref. ${ }^{62}$ ).

\section{[H1] Ideal blueprint nets as design targets}

\section{[H2] Edge-transitive nets and their derived nets}

The minimal transitivity principle states that the underlying nets of MOFs and COFs are more likely to be nets of minimal transitivity. Indeed, topological analysis of MOFs reveals the common occurrence of underlying nets with minimal transitivity. The minimal number of edge types for uninodal and binodal nets is one, and for nets with three types of nodes is two. Edgetransitive nets - nets with only one type of edge - are the central nets for MOF design 
because they are the most suitable blueprints and reliable design targets, and a steppingstone to more intricate nets.

The number of nets with one type of edge is infinite if there is no defined constraint. Accordingly, the most crucial and challenging step during the design process is to constrain the number of possibilities, and the limited number of edge-transitive nets makes them suitable targets in crystal chemistry. There are 96 three-periodic nets with only one type of edge currently listed in the RCSR database, but only 53 are 'basic' edge-transitive nets. Of the 96 nets, 13 are binary versions of the basic edge-transitive nets, and the spn-z net is a spn net with a special conformation. Here, we use two constraints to analyse the remaining 82 nets, some of which can be regarded as derivatives of basic edge-transitive nets. The first constraint is full symmetry embedding, which means that the distance between unconnected nodes should not be smaller than the edge length. Without this constraint, the number of nets with one type of edge will be infinite for the unlimited intersecting edges of same kind of node as those in basic edge-transitive nets. At present, there are 17 edge-transitive nets with intersecting edges listed in the RCSR database. The second constraint is no multiple edges or collisions, that is, two or more edges or nodes that share the same neighbouring nodes. Currently, there are 12 nets of this type, which are termed noncrystallographic, in the RCSR database ${ }^{127}$. MOF design based on these collisional nets has not been widely explored. Thus, we do not further elaborate on their potential for design, although these nets could be suitable targets if appropriate design methodologies are employed. With these two constraints, there are 53 known three-periodic basic edge-transitive nets and five two-periodic edge-transitive nets. In the following, we use edge-transitive nets to refer to basic edge-transitive nets suitable for the practice of reticular chemistry. These edge-transitive nets and their coordination numbers are summarized in Table 1. The coordination figure describes the geometry of vertex coordination. In this Review, we use the RCSR abbreviations of the coordination figures ${ }^{128}$, which are also listed in the footnote of Table 1. Nets can be denoted in terms of the coordination figures; for example, SO denotes the soc net, where $\mathrm{S}$ and $\mathrm{O}$ are the symbols for the square and octahedral coordination figures, respectively.

Replacing the nodes in edge-transitive nets with groups of nodes, produces more intricate variants, such as derived nets ${ }^{129}$ and related nets ${ }^{130}$. Thus, the initial edge-transitive nets are termed parent nets. MOFs with linkers featuring two or more branch points can express different underlying nets associated with the same parent net. For the topological analysis of a MOF with branched ligands, both the underlying derived net and the parent net provide useful information. The derived net gives the complete topological information because each branch 
point of a linker is regarded as a node of the resultant net. In terms of the design process, the parent nets, especially edge-transitive parent nets, are more reliable design targets. The difference between derived and related nets is the coordination number of the nodes that have not been replaced. If the coordination number of these unreplaced nodes is unchanged, the new net is a derived net. If the coordination number of unreplaced nodes increases, the new net is a related net ${ }^{128}$. The vertex coordination figure of a given net therefore dictates the requisite geometrical information of the building units to be transplanted in the chemical building blocks for the successful practice of reticular chemistry. Accessing building units that code a specific net is key for the design of MOFs, and we term these building units net-coded building units (net-cBUs).

The net-cBUs of a selected net are building units or sets of linked building units that embed the unique geometrical code of that net, enclosing the essential information for selecting the appropriate building blocks during the rational design of MOFs. Accordingly, the net-cBUs of a net include all the SBUs as well as the zero-periodic, one-periodic and two-periodic sets of connected SBUs, such as tertiary building units (TBUs), SBBs and SBLs. Multiple nets can share similar basic SBUs (Fig. 5), and thus these building units are not exclusive for the reticulation of a single net, suggesting that these building units are dormant and need additional coded information to be expressed building units for that given net.

To provide a design toolbox for the practice of reticular chemistry, we analyse the 58 edgetransitive nets (53 three-periodic nets and five two-periodic nets) based on their associated basic building units and their classification as dormant net-cBUs or expressed net-cBUs. The 58 edge-transitive nets can be categorized into three types (Fig. 5). Type I nets contain unique coordination figures, proffering only one edge-transitive target for the associated net-cBU. For type I nets, the success rate for MOF design will be high given the ready access to the expressed net-cBUs for a given net. Type II nets contain coordination figures shared by two or three nets. Therefore, the additional coded information unique to a given net embedded in the connectivity of its dormant net-cBUs, such as the torsion angle between nodes and the distortion angle of nodes, needs to be defined to express the dormant net-cBUs. Type III nets contain coordination figures shared by seven nets and are based on linking only square or tetrahedral building units. Note that although MOFs based on dia and nbo nets are commonly observed, their respective building units are not exclusive to these nets only(Fig. 5). Therefore, we have opted to classify them as type III. With the exception of dia and nbo nets, we sense that the success rate of MOF design based on type III nets and involving only direct linking of the SBUs will be lower than for type I and type II nets. Nevertheless, the design of MOFs based on type III nets is still 
plausible by introducing more coded information for the proper connectivity of the associated dormant net-cBUs ${ }^{98}$. In the following, we give examples of MOFs based on these threeperiodic edge-transitive nets or their derived nets for 49 of the 53 three-periodic edge-transitive nets (MOF examples of the ssc, ifi, thp and mgc nets were not found) to expound how building blocks link to real structures based on these nets.

In certain nets, the building units are often elongated, compressed or distorted from the regular polyhedron. The geometrical information of the building units is also useful for structure design. Here, we use the vertex-centre-vertex angle to distinguish similar building units. The vertex-centre-vertex angle in regular tetrahedral, octahedral, cubic and cuboctahedral building units is $109.5^{\circ}, 90^{\circ}, 70.5^{\circ}$ and $60^{\circ}$, respectively (Fig. 6a).

[H3] Type I edge-transitive nets. Type I nets are based on linking expressed net-cBUs. Therefore, connecting building blocks with suitable geometrical information will probably give the designed structure. The only $3 \mathrm{H}$ edge-transitive net is a two-periodic kgd net, which is also the only binodal two-periodic edge-transitive net. The SO net soc has been well explored with 6-c trinuclear clusters and branched 4-c linkers ${ }^{34,35,41}$. MOFs based on the SH net she can be constructed from 6-c hexanuclear Zr clusters and 4-c tetratopic porphyrin-based linkers, as in PCN-224 (ref. ${ }^{131}$ ). she MOFs can also be constructed from 4-c $\mathrm{Cu}$ paddlewheels and 6-c hexatopic planar linkers, such as in she-MOF-1 to she-MOF-4 (ref. ${ }^{132}$ ). The only $(4,6)-c$ SP net is the stp net. One example of a stp-MOF is MOF-892, built from $\mathrm{Zr}$ clusters that express a trigonal prismatic building unit, and the $1^{\prime}, 2^{\prime}, 3^{\prime}, 4^{\prime}, 5^{\prime}, 6^{\prime}$-hexakis(4-carboxyphenyl)benzene linker, which represents a rectangular building unit ${ }^{133}$. We did not find a MOF example of the $(4,6)$-c ifi net. Although tetrahedral and trigonal prismatic building units are common, the highly elongated prismatic building unit and the distorted tetrahedral building unit make it difficult to obtain MOFs based on the ifi net (Fig. 6b). The angle between two adjacent edges from each base of the prism in the ifi net is as large as $135.6^{\circ}$. In comparison, the angle within the prism in the stp net is $70.5^{\circ}$, which is more achievable with an easily obtained 6-c trinuclear cluster. The angles within the tetrahedral building unit are also distorted to $81.8^{\circ}$ and $135.6^{\circ}$. The only $(6,6)-c$ net is the nia net, which is based on a 6-c octahedron and 6-c trigonal prism. Representative examples of nia-MOFs were built from trigonal prismatic trinuclear clusters (In for JUC-101 (ref. ${ }^{40}$ ), Mn for JUC-102 (ref. ${ }^{40}$ ) and Yb for UTSA-62 (ref. ${ }^{134}$ )) and octahedral organic linkers. We did not find a MOF example based on the 8-c thp net. Although the thp net contains only one type of node and one type of edge, the coordination figure is a distorted bisdisphenoid, making it difficult to design a thp-MOF structure (Fig. 6c). In the bisdisphenoid 
of the thp net, there are three different angles between adjacent nodes: $60^{\circ}, 73.4^{\circ}$ and $94.1^{\circ}$. The $60^{\circ}$ angle means that all nodes are linked to regular triangles, and the thp net can be regarded as regular triangles linked by shared nodes. We believe that a non-linear organic linker with appropriate rotation, torsion and translation will be the key to obtaining thp-MOFs. The only edge-transitive net with trigonal and cubic coordination figures is the $(3,8)$-c the net. An early example of a the-MOF was reported with tetranuclear cuboid clusters and tritopic linkers $^{54}$, and $\mathrm{Zr}$-the-MOFs are obtained by linking hexanuclear $\mathrm{Zr}$ clusters and twisted tritopic linkers ${ }^{135,136}$. The flu net is the only $(4,8)$-c edge-transitive net with tetrahedral and cubic coordination figures. Zr-flu-MOFs are formed by linking hexanuclear clusters and tetratopic tetrahedral linkers ${ }^{60,137}$. Although the flu net has cubic symmetry, the flu-MOFs constructed from hexanuclear clusters have tetragonal symmetry as the cluster adopts a cuboid geometry. Thus, the tetrahedral building units are distorted tetrahedra with $90^{\circ}$ and $120^{\circ}$ angles. The ocu net is the only $(6,8)$-c edge-transitive net with octahedral and cubic coordination figures. One example of an ocu-MOF is based on linking 6-c trinuclear Zn clusters and 8-c Zn SBBs ${ }^{138}$. The underlying net of the whole structure is the ocu-derived $(3,4,6)$-c lwh net.

All highly coordinated edge-transitive nets (a total of nine nets with a highest coordination number of $\geq 12$ ) are reticulated by unique building unit combinations. Two nets containing cuboctahedral building units are the 12-c fcu f7,58,68,75,76,139 and $(4,12)$-c ftw $^{59,71,140,141}$ nets, which have been well explored using hexanuclear clusters ( $\mathrm{Zr}$, rare earth) and octanuclear clusters $(\mathrm{Ni}, \mathrm{Co})$. Two nets containing truncated tetrahedral building units are the $(3,12)$-c ttt and $(6,12)$-c mgc nets. One example of a ttt-MOF is based on decanuclear $\mathrm{Zn}$ clusters and a bent truxene-tribenzoate linker $\left(5,5^{\prime}, 10,10^{\prime}, 15,15^{\prime} \text {-hexaethyltruxene-2,7,12-tribenzoic acid }\right)^{142}$. We did not find an example of a mgc-MOF, which might be due to two factors. First, the 12-c truncated tetrahedral building unit is less common than the 12-c cuboctahedral building unit. Second, the hexagonal building units in the mgc net are not regular hexagons but adopt a chair conformation (Fig. 6d). Two nets containing hexagonal prismatic building units are the $(4,12)$ c shp net and (6,12)-c alb net. Reported shp-MOFs are constructed by linking hexanuclear $\mathrm{Zr}$ clusters $^{143}$, nonanuclear rare-earth clusters ${ }^{9,144}$ or octadecanuclear Np clusters ${ }^{145}$ to 4 -c organic linkers, or by linking $\mathrm{Cu}$ paddlewheel clusters to 12 -c branched organic linkers ${ }^{9}$. The alb-MOF can be obtained by linking rare-earth clusters ${ }^{9}$ or $\mathrm{Zr}$ clusters ${ }^{146}$ to 6 -c trigonal prismatic organic linkers. The only net containing icosahedral building units is the $(4,12)$-c ith net, with 4-c tetrahedra. An ith-MOF, MOF-812, was obtained by linking hexanuclear $\mathrm{Zr}$ clusters and tetrahedral linkers with four carboxylate groups acting as monodentate ligands ${ }^{60}$. Only two nets have a highest coordination number of 24: the $(3,24)$-c $3 R$ net rht and the $(4,24)$-c TK net twf. 
It remains challenging to reliably obtain structures based on 24-c metal clusters as MBBs. As an alternative, these platforms have been explored with the SBB approach based on edgetransitive derived nets. The rht-MOFs have been widely explored with 24-c

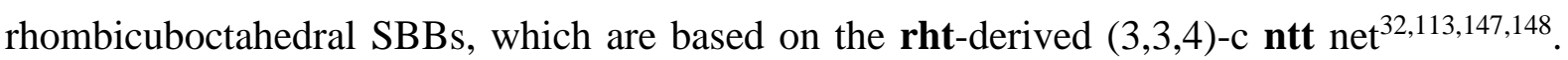
One example of a twf-MOF is JLU-Liu-40, which is constructed from two types of Zn node (square and tetrahedral) and in-plane rotated tetratopic organic linkers ${ }^{149}$. The unique $144^{\circ}$ angle of the triazole centre in the 5,5-(1H-1,2,4-triazole3,5-diyl) diisophthalate linker enabled the formation of 24-c truncated octahedral SBBs.

[H3] Type II edge-transitive nets with trigonal building units. For type II nets, more detailed geometry information is needed to distinguish the multiple nets with the same coordination figures for the successful design of a targeted net. Both the three-periodic srs net and twoperiodic hcb net are based on linking trigonal building units. The dihedral angle between two adjacent trigonal planes has a key role in directing the formation of a particular structure. The srs net exhibits a $70.5^{\circ}$ (or $109.5^{\circ}$ considering the reverse side) torsion angle for each extended trigonal plane, whereas in the planar hcb net, this angle is $0^{\circ}$. One representative srs-MOF is a Zn-MOF formed in a two-step synthesis ${ }^{150}$. The MOF-5-type pcu-MOF, based on tetranuclear $\mathrm{Zn}$ clusters and a pyrazole carboxylate linker, undergoes an elimination reaction in water. The elimination of metal ions and organic linkers is ordered, creating ordered vacancies within the initial MOF. The new MOFs are based on the underlying srs net, illustrating that the pcu net can be regarded as two merged srs nets. Another notable srs-MOF is an $\mathrm{Ag}-\mathrm{MOF}$ with a tritopic imidazole linker bridging 2-c $\mathrm{Ag}^{+}$ions ${ }^{151}$. This MOF exhibits a unique 54-fold interpenetrated structure, which is the highest interpenetration number among all MOFs.

[H3] Type II edge-transitive nets with coordination figure $3 S$ and $3 T$. The $(3,4)-c$ tbo and pto nets are based on linking trigonal and square building units. There are two notable differences between tbo and pto nets. First, they have square building units of different geometries. In the tbo net, the 4-c building unit is a rectangle with a ratio of $\sim 0.707$ between the short edge and the long edge, whereas in the pto net, the 4-c building unit is a regular square. Second, the dihedral angle between the connected building units differs: the torsion angle between adjacent squares and triangles is $90^{\circ}$ in the tbo net but $\sim 54.7^{\circ}$ in the pto net. This difference in the torsion angle explains the difference in the associated MOF structures. When a $\mathrm{Cu}$ paddlewheel cluster is used as the 4-c building block, a planar tritopic linker gives tbo- 
MOFs as it meets the requirement for a $90^{\circ}$ torsion angle ${ }^{152}$. A small twist of the planar tritopic organic linkers can promote the construction of pto-MOFs instead of tbo-MOFs ${ }^{152}$. The $(3,4)-$ c ctn and bor nets are based on linking trigonal and tetrahedral building units, but differ in the geometries of their tetrahedral building units. In the ctn net, the tetrahedral building unit is a regular tetrahedron, whereas in the bor net, the tetrahedral building unit is distorted with angles of $70.5^{\circ}$ and $131.8^{\circ}$. Using geometrically flexible single-metal tetrahedral nodes $\left(\mathrm{Cu}^{+}\right)$and freely rotating tri-pyridine-based linkers, both bor and ctn nets can form from the same building blocks ${ }^{153,154}$. The bor and tbo nets are also ideal platforms for the construction of pillared MOFs based on the SBL approach ${ }^{92}$.

[H3] Type II edge-transitive nets with coordination figure ST. The pts and pth nets are both based on linking square and tetrahedral building units. However, the geometries of the respective tetrahedral building units differ: the building unit of the pts net is a regular tetrahedron, whereas that of the pth net is a distorted tetrahedron with angles of $90^{\circ}, 109.5^{\circ}$ and $131.8^{\circ}$, making pth-MOFs more difficult to obtain with high-symmetry tetrahedral building blocks. The pts platform has been widely explored with paddlewheel clusters and tetrahedral organic linkers since the first pts-MOF, MOF-11, was reported ${ }^{155}$. The construction of a pth-MOF necessitates more control of the linker angles. For example, CMOF-1 is based on the underlying pth-derived $(3,4)$-c phw net, constructed from paddlewheel clusters and tetratopic linkers with angles $\left(94.4^{\circ}, 101.3^{\circ}\right.$, and $\left.136.8^{\circ}\right)$ constrained by a $\mathrm{PO}_{4}$ group ${ }^{156}$.

[H3] Type II edge-transitive nets with coordination figure $H, O$ and $P$. There are three typical 6-c building units found in edge-transitive nets: hexagonal, octahedral and trigonal prismatic. Two edge-transitive nets, the three-periodic hxg net and the two-periodic hxl net, are based on linking only hexagonal building units but differ in the dihedral angle between two adjacent hexagonal planes. In the hxg net, there is a $70.5^{\circ}$ (or $109.5^{\circ}$ considering the reverse side) torsion angle for each extended hexagonal plane, while in planar hxl net, this angle is $0^{\circ}$. This torsion angle was an important consideration in the design of a pbz-MOF (where pbz is hxg-a $)^{63}$. Using a hexagonal hexanuclear $\mathrm{Zr}$ cluster as the inorganic building unit, a hexagonal 6-c organic linker was used to provide the $70.5^{\circ}$ dihedral angle found in the pbz structure.

Three edge-transitive nets, pcu, bcs and crs, are based on linking only octahedral building units but differ in the geometries of these units. Unlike the regular octahedra in the pcu net ${ }^{47-}$ 49 , the octahedra in the bcs and crs nets are distorted. In the bcs net, the octahedra are compressed to trigonal antiprisms with angles of $70.5^{\circ}$ and $109.5^{\circ}$, corresponding to cubes with 
two opposite nodes missing. In the crs net, the octahedra are elongated to trigonal antiprisms with angles of $120^{\circ}$ and $60^{\circ}$, corresponding to cuboctahedra with six planar nodes missing. By using the same tetranuclear Zn clusters as in MOF-5 (pcu), but distortedly linking the clusters, a bcs-MOF was reported with the bulky 9,10 -anthracenedicarboxylate linker ${ }^{157}$. MOF-500 is an example of a crs-MOF ${ }^{158}$, constructed from trinuclear Fe clusters, the BPDC linker and the cis-1,2-bis-4-pyridylethane linker.

Two edge-transitive nets, acs and lcy, are based on linking only trigonal prismatic building units. In the acs net, the angles of the trigonal prisms are $70.5^{\circ}$ and $90^{\circ}$. In comparison, in the lcy net, the trigonal prisms are twisted and compressed, with angles of $60^{\circ}$ and $99.5^{\circ}$. MIL-88 is an example of an acs-MOF and contains 6-c trinuclear clusters and a ditopic linker ${ }^{36}$. An acs-MOF with 6-c trinuclear clusters and a 6-c triptycene-based organic ligand has also been reported $^{159,160}$. The trigonal prismatic building units in the lcy net can be morphed into octahedral building units connected by bent edges. Using a tetranuclear Zn cluster, an lcy-MOF was reported by replacing the linear linker 1,4-BDC in MOF-5 (pcu-MOF) with the bent linker $1,3-\mathrm{BDC}^{161}$.

[H3] Type II edge-transitive nets with coordination figure $3 O$ and TO. The pyr and spn nets are $(3,6)$-c nets based on linking trigonal and geometrically different octahedral building units. The octahedra in the pyr net are slightly distorted, with angles of $80.4^{\circ}$ and $99.6^{\circ}$, whereas the octahedra in the spn net are elongated to trigonal antiprisms with angles of $146.4^{\circ}$ and $33.6^{\circ}$. One representative example of a pyr-MOF is MOF-150, which contains 6-c tetranuclear $\mathrm{Zn}$ clusters and tritopic linkers ${ }^{162}$. Examples of Zr-spn-MOFs include MOF-808 (ref. ${ }^{60}$ ), PCN-777 $\left(\right.$ ref. $^{64}$ ) and MOF-818 (ref. ${ }^{65}$ ), in which hexanuclear $\mathrm{Zr}$ clusters are linked with tritopic planar linkers or clusters. The only reported RE-spn-MOF is Tb-spn-MOF-1, based on the 5-(4H1,2,4-triazol-4-yl)isophthalate linker ${ }^{10}$.

The gar, toc and iac nets are (4,6)-c nets based on linking tetrahedral and octahedral building units. In the gar net, the tetrahedra are distorted with angles of $101.5^{\circ}$ and $126.9^{\circ}$, and the octahedra are compressed to trigonal antiprisms with angles of $66.4^{\circ}$ and $113.6^{\circ}$. In the toc net, the tetrahedra are regular, while the octahedra are compressed to trigonal antiprisms with angles of $70.5^{\circ}$ and $109.5^{\circ}$ (the same as those in the bcs net octahedra). In the iac net, the tetrahedra are the same as those in the gar net, but the octahedra are elongated with angles of $113.6^{\circ}$ and $66.4^{\circ}$. An example of a gar-MOF is $\mathrm{ZIF}-5$, which contains both $\mathrm{In}^{3+}$ and $\mathrm{Zn}^{2+}$ nodes $^{163}$. The gar-MOF has also been reported with hexanuclear $\mathrm{Zr}$ clusters and tetratopic calixarene-based linkers ${ }^{164}$. A mixed-metal MOF, BUT-52, is an example of a toc-MOF, and 
contains $\operatorname{In}\left(\mathrm{O}_{2} \mathrm{C}-\right)_{4}$ as the 4-c building unit and $\mathrm{Cu}_{6} \mathrm{~S}_{6}$ as the 6-c building unit ${ }^{165}$. The ditopic linker 6-mecaptopyridine-3-carboxylate was formed in situ from 6,6'-dithiodinicotinic acid by breaking the $\mathrm{S}-\mathrm{S}$ bond during a solvothermal reaction. One example of an iac-MOF is formed by linking tetranuclear $\mathrm{Zn}$ clusters and elongated branched tetracarboxylate organic linkers, based on the underlying iac-derived $(3,6)$-c act net ${ }^{166}$. The building units in the derived act net (regular octahedra and elongated tetrahedra) are morphed from the parent iac net.

[H3] Type II edge-transitive nets with coordination figure $C$ and SC. The bcu and reo nets are 8-c nets based on linking only cubic building units. In the bcu net, the cubes are regular, while in the reo net, the cubes are elongated to cuboids with angles of $60^{\circ}$ and $120^{\circ}$, corresponding to cuboctahedra with four planar nodes missing. The Zr-bcu-MOFs were obtained by replacing some of the linear linkers used in fcu-MOFs with ditopic linkers of different geometries, such as twisted linkers ${ }^{167}$ or zigzag linkers ${ }^{101}$. When a planar bent linker was used, reo-MOFs, such as DUT-51 (ref. ${ }^{93}$ ) and JLU-MOF58 (ref. ${ }^{94}$ ), were obtained. Geometrically, this type of bent linker is not a necessary condition for Zr-reo-MOFs, but it excludes the formation of fcu-MOFs.

The (4,8)-c scu, csq and sqc nets are based on linking square and cubic building units. The scu net contains regular cubes and distorted squares with angles of $70.5^{\circ}$ and $109.5^{\circ}$; the $\mathbf{c s q}$ net contains distorted cubes with angles of $48.2^{\circ}, 70.5^{\circ}$ and $90^{\circ}$, and the distorted squares; and the sqc net contains even more distorted cubes and squares. Zr scu-MOFs ${ }^{168}$, $\mathbf{c s q - M O F s}{ }^{140,169-}$ ${ }^{171}$ and sqc-MOFs ${ }^{172}$ can be obtained with twisted planar tetratopic linkers.

[H3] Type III edge-transitive nets. For type III nets, structural design requires strict control of the geometry. There are seven nets linking square building units: three-periodic [11] nets (nbo, lvt and rhr), three-periodic [21] nets (ssa and ssb) and two-periodic [11] nets (sql and kgm). The nbo-MOF was first reported with a $\mathrm{Cu}$ paddlewheel and twisted BDC linkers ${ }^{98}$, which excluded the formation of two-periodic sql-MOFs. The lvt-MOFs can be obtained from hexanuclear $\mathrm{Zr}$ clusters and the planar elongated tetratopic linker [1,1':4',1"]-terphenyl$3,3^{\prime \prime}, 5,5^{\prime \prime}$-tetracarboxylate ${ }^{66,67}$. One example of a rhr-MOF is BUT-41, comprising square $\mathrm{Ni}^{2+}$ nodes and tetratopic pyrazole linkers ${ }^{173}$. The [21] nets ssa and ssb differ in terms of the pillared layer: kgm for ssa, and sql for ssb. One example of an ssa-MOF is ZJU-25, constructed from $\mathrm{Cu}$ paddlewheels and out-of-plane bent tetratopic linkers, based on the underlying ssa-derived $(3,4)$-c sty net ${ }^{174}$. The ssb-MOF has been reported with $\mathrm{Cu}$ paddlewheels and bulky linkers in 
NOTT-109 (ref. ${ }^{175}$ ) and MMPF-1 (ref. ${ }^{176}$ ), based on an ssb-derived net $(3,4)$-c stx, or flexible rotated linkers in DUT-12 (ref. ${ }^{177}$ ), based on another ssb-derived net, $(3,4)$-c stu.

The seven nets with tetrahedral building units are all three-periodic nets, namely the [11] nets (dia, lcs, lcv, qtz, sod and ana) and the [21] net ssc. As it is widely observed, the dia net is the most commonly explored three-periodic COF platform ${ }^{4}$. The sod and ana nets correspond to the zeolitic topologies SOD and ANA, respectively, which are ideal targets for the construction of ZIFs ${ }^{163}$ and ZMOFs ${ }^{11}$. Examples of ana-MOFs include ZIF-14 (ref. ${ }^{12}$ ) and ana-ZMOFs (ref. ${ }^{178}$ ). Examples of Ics-MOFs include ZIF-72 (ref. ${ }^{12}$ ) and bio-MOF-100 to bioMOF-103 (refs ${ }^{179,180}$ ). One example of a qtz-MOF was obtained from an In salt and chiral camphoric acid ${ }^{181}$. The $\mathrm{P}$ helix and $\mathrm{M}$ helix structures are obtained from D-camphorate acid and L-camphorate, respectively. One example of an lcv-MOF is $\mathrm{Ag}_{2}\left(\mathrm{Me}_{4} \mathrm{bpz}\right)$, constructed from $\mathrm{Ag}^{+}$nodes and bipyrazole (bpz) linkers ${ }^{182}$.

[H3] Dormant and expressed net-coded building units. For type II and type III nets, more information on the linking of dormant net-cBUs is needed to attain the requisite expressed netcBUs, suggesting the possibility of various plausible design routes to express dormant netcBUs for the construction of a specific MOF. Here, we use the tbo net as an example to show that there are at least four interpretations for the design of tbo-MOFs (Fig. 7a). The tbo net contains two types of SBU: a trigonal SBU and a square SBU. These two SBUs are also tbocBUs. In the first approach, a tbo-MOF can be designed by straightforwardly linking two types of building blocks that geometrically match the SBUs. These SBUs afford a $90^{\circ}$ torsion angle between the adjacent square and triangle and do not promote the pto net with a torsion angle of $\sim 54.7^{\circ}$ (ref. ${ }^{152}$ ) (Fig. 7a). Second, connected SBUs can be grouped into diverse combinations. Linking four trigonal SBUs and one square SBU gives a zero-periodic branched TBU with a cuboid geometry as the tbo-cBU. A tbo-MOF can thus be designed by linking square building blocks to branched octatopic building blocks (typically organic linkers) that geometrically match the TBU $118,122,123$. Third, linking four trigonal SBUs and six square SBUs gives a zeroperiodic super ada polyhedron as the tbo-cBU. Therefore, a tbo-MOF can be designed by linking the pre-selected tet-SBBs via node-sharing, which corresponds to assembly through the SBB approach. Last, the SBUs can also link to infinite two-periodic SBLs. For the tbo net, linking square SBUs and their adjacent trigonal SBUs gives sql-1 layers as tbo-cBUs. A tboMOF can then be designed by pillaring the pre-selected sql SBLs with square SBUs ${ }^{118,122,123}$. For each design approach, the tbo-cBUs provide all the required geometrical information about the requisite building blocks. The last three design routes have a higher success rate than the 
first, because the direct combination of the two building units can give multiple results (for example, the edge-transitive pto net). We thus refer to the building units in the first route as dormant net-cBUs and the building units in other routes as expressed net-cBUs.

The torsion angles between adjacent building units can also help to transform dormant netcBUs into expressed net-cBUs. For example, the hexagonal SBU is a dormant net-cBU because it affords multiple edge-transitive nets (hxl and hxg). In this case, if the specific information of the torsion angle is coded into the selected building units, the hexagonal SBU can be transformed into an expressed hxl ( $0^{\circ}$ twist) or hxg $\left(70.53^{\circ}\right.$ twist $)$ net-cBU (Fig. $\left.7 b\right)$.

\section{[H2] Merged net platforms.}

The merged net approach is the latest addition to the design toolbox in reticular chemistry and enables the design of intricate multi-component structures ${ }^{10}$. This approach provides a rationale for interpreting mixed-linker structures based on merging two edge-transitive nets into a minimal edge-transitive net, in which one ligand makes one net, and the second ligand makes the other (Fig. 8). Both ligands share the same inorganic MBB. We illustrate this concept with the sph net, which encloses two edges generated by merging two edge-transitive nets, namely $(3,6)$-c spn and 6-c hxg. The resultant merged net is a $(3,6,12)$-c sph net with net transitivity [32] and encloses three nodes and two distinct edges. This merged net offers the potential for the design of intricate mixed-linker MOFs, sph-MOF-1 to sph-MOF-4, which contain hexanuclear rare-earth clusters, 3-c tritopic linkers and larger 6-c hexatopic linkers ${ }^{10}$ (Fig. 8d). The 6-c hexatopic linker can be formed from one hexatopic linker or two tritopic linkers. Connecting hexanuclear clusters and 3-c organic linkers forms spn frameworks, whereas connecting hexanuclear clusters and 6-c organic linkers forms $\mathbf{h x g}$ frameworks. The resultant sph-MOFs are the first examples of MOFs in which the underlying net is merged from two three-periodic edge-transitive nets. sph-MOF-3 is the first mixed-linker MOF to enclose both trigonal and hexagonal linkers. The merged net approach was also applied to construct a mixed-linker mesoporous MOF, sph-MOF-4.

Nets such as sph are termed 'three-periodic and three-periodic' ( $3 p-3 p)$ merged nets as both parent nets are three-periodic nets. Another $3 p-3 p$ merged net is the $(4,12)$-c tam net, which is merged from the 6-c pcu net and the (4,6)-c she net ${ }^{183}$. One reported tam-MOF is PCN-201, constructed from 12-c hexanuclear clusters, 4-c porphyrin-based linkers and 2-c $\mathrm{Cu}-$ isonicotinate moieties ${ }^{183}$. Merged nets can also be based on merging three-periodic and twoperiodic nets $(3 p-2 p)$, three-periodic and one-periodic nets $(3 p-1 p)$ and three-periodic and zero-periodic nets ( $3 \mathrm{p}-0 \mathrm{p})$. In the following, we analyse representative mixed-linker MOFs to 
show the power of merged nets for understanding the underlying principles of multi-component structures.

One example of a $3 p-0 p$ merged net is the three-periodic $9-c$ ncb net, which is merged from a three-periodic 6-c pcu net and a zero-periodic 3-c tet net (a tetrahedral cage ${ }^{42-45}$. Reported ncb MOFs are constructed from 9-c trinuclear clusters and two types of organic linker: a ditopic carboxylate linker (L1) and a ditopic pyridine-carboxylate linker (L2) in a molar ratio 1:2. Each cluster connects to six carboxylates (three from L1 and three from L2) and three pyridines (from L2). The connections between the clusters and L1 form metal-organic tetrahedra based on the underlying 3-c tet net. The connections between the clusters and L2 form three-periodic frameworks with the underlying 6-c pcu net. Merging these two frameworks leads to threeperiodic 9-c ncb frameworks (Fig. 8a).

The $(3,6)$-c ith-d platform is a $3 \mathrm{p}-1 \mathrm{p}$ merged net, in which a three-periodic pto net and oneperiodic lcw net are merged. $(3,6)$-c ith-d is one of the most explored merged net platforms, and can be synthesized by linking tetranuclear Zn clusters with both tritopic and ditopic carboxylate linkers ${ }^{184-188}$ (Fig. 8b). Connections between the clusters and tritopic linkers form the three-periodic pto frameworks, while connections between the clusters and ditopic linkers form the one-periodic lcw rods. Merging these two parts leads to three-periodic ith-d frameworks. DUT-60, an ith-d MOF, has the highest accessible pore volume $\left(5.02 \mathrm{~cm}^{3} \mathrm{~g}^{-1}\right)$ of all reported MOFs and $\mathrm{COFs}^{189}$. The ith-d platform can also be obtained using paddlewheels, tritopic carboxylate linkers and ditopic pyridine linkers ${ }^{190,191}$. Another example of a $3 p-1 p$ merged net is $(3,6)$-c muo, based on merging a three-periodic $(3,4)$-c sln net and one-periodic 2-c zigzag chain. In UMCM-1, octahedral hexanuclear Zn clusters link to two organic linkers, and the molar ratio between tritopic BTB linkers and ditopic BDC linkers is 4:3 (ref. ${ }^{192}$ ). Connections between the clusters and BTB linkers form the three-periodic sln framework, while connections between the clusters and BDC linkers form the one-periodic zigzag chain. Merging these two parts leads to a three-periodic muo framework.

One example of a $3 p-2 p$ merged net MOF is based on a $(3,9)-c$ net, which is merged from a three-periodic 6-c acs net and a two-periodic 3-c hcb net (Fig. 8c). CPM-230 (ref. ${ }^{46}$ ) contains 9-c trinuclear clusters, ditopic carboxylic linkers and tritopic pyridine linkers. The connections between the clusters and ditopic linkers form the acs framework, while the connections of the clusters and tritopic pyridine linkers form a two-periodic hcb framework. Another example is MOF-520-BPDC, based on the (3,16)-c skl net, which merges the three-periodic $(3,12)$-c fon net and two-periodic 4-c sql net ${ }^{72}$. MOF-520-BPDC contains octanuclear Al clusters, tritopic BTB linkers and ditopic BPDC linkers. The connections between the clusters and BTB linkers 
form a three-periodic fon framework, while the connections of the clusters and BPDC linkers form a two-periodic sql framework. MOF-1210 based on the $(4,4,12)$-c $\mathbf{p f m}$ net, merges the three-periodic (4,8)-c flu net and two-periodic 4-c sql net ${ }^{193}$. MOF-1210 contains hexanuclear $\mathrm{Zr}$ clusters, tetrahedral single-atom $\mathrm{Cu}(\mathrm{I}) \mathrm{MBBs}$, square planar $\mathrm{Cu}$ (II) $\mathrm{MBBs}$ and ditopic isonicotinic acid linkers. The connections between the $\mathrm{Zr}_{6}$ clusters, $\mathrm{Cu}(\mathrm{I}) \mathrm{MBBs}$ and isonicotinic acid form a three-periodic flu framework, while the connections of the $\mathrm{Zr}_{6}$ clusters, $\mathrm{Cu}$ (II) MBBs and isonicotinic acid form a two-periodic sql framework.

The total number of even the simplest merged nets, nets based on merging two edgetransitive nets, remains unclear. A more extensive and systematic library of merged nets will assist the design of intricate MOFs based on mixed ligands. The enumeration of $3 p-3 p, 3 p-2 p$, $3 p-1 p$ and $3 p-0 p$ merged nets could lead new and more intricate platforms.

A systematic analysis of multi-component structures ${ }^{194-196}$ using the principles of the merging concept could provide topological insight into their assembly and further understanding towards their rational construction. Some MOFs are explored for their ability to accommodate additional moieties within their pore system, affording further bridging of existing MBBs through a process referred to as linker installation ${ }^{167}$ or partition ${ }^{197,198}$. The added moieties form a periodic structure (typically zero-periodic or two-periodic), and thus the resultant MOF structures can be regarded as connected periodic structures, suggesting a plausible topological relationship between the two merged nets. Similarly, removing organic or inorganic MBBs from a given MOF, while maintaining the structural connectivity in three dimensions, can generate periodic defects or ordered vacancies ${ }^{195,196}$; a process we term topologically reversed merging.

\section{[H2] Important non-edge-transitive platforms}

If a net is commonly observed in reported structures, and is isoreticular designable, it can generally be regarded as a promising outlier to be explored as a blueprint for design. Accordingly, nets such as $(3,6)-\mathrm{c}$ qom and 4-c mtn, although not directly derived from edgetransitive nets, warrant further exploration. The first qom-MOF, MOF-177, was constructed from tetranuclear Zn clusters and tritopic BTB linkers ${ }^{199}$. The qom-MOF platform has been explored isoreticularly with various expanded ligands ${ }^{184}$. In another example, the formation of $(3,6)$-c qom, pyr and rtl structures was controlled by varying the position of the functional groups on the BTB linkers ${ }^{200}$. The first mtn-MOFs, MIL-100 (ref. ${ }^{201}$ ) and MIL-101 (ref. ${ }^{37}$ ), 
comprised supertetrahedral building blocks, constructed from trinuclear clusters and tritopic BTC linkers in MIL-100, based on the underlying moo net, or ditopic BDC linkers in MIL101, based on the underlying mtn-e net. mtn-MOFs with larger cavities were reported with extended tritopic building blocks, such as benzo-tris-thiophene carboxylate in PCN-332 (ref. ${ }^{38}$ ), triazine-1,3,5-tribenzoate in PCN-333 (ref. ${ }^{38}$ ) and trinuclear Cu clusters linked by $1 H$-pyrazole4-carboxylate in MOF-919 (ref. ${ }^{65}$ ). Another mtn-MOF constructed using a similar SBB approach is based on supertetrahedral building blocks formed from tetranuclear Tb clusters and triazine-1,3,5-tribenzoate linkers ${ }^{202}$. Moreover, single-metal-ion MBBs have also been used to build an mtn-MOF, constructed from 4-c hexamethylenetetramine linkers and bridging 2-c $\mathrm{Cd}^{2+}$ ions $^{203}$.

\section{[H1] Isoreticular design strategies}

\section{[H2] Building block modification and substitution}

The modification of building blocks is the most widely used method to introduce different functional groups into MOFs. Functional groups can be introduced before ${ }^{204}$ or after $^{205}$ material assembly.

Pre-designed functional groups can be introduced into organic linkers before material synthesis for targeted applications. For example, the thiadiazole group in a ditopic linear carboxylic ligand was functionalized to afford the $\pi$-conjugated and electron-deficient $4,4^{\prime}-$ (benzoic]i1,2,5]thiadiazole-4,7-diyl) dibenzoic acid ligand ${ }^{204}$. The assembly of this linker and a hexanucler Zr cluster yields a luminescent Zr-BTDB-fcu-MOF with an ultralow amine detection limit ${ }^{204}$. A grafted group can also contract the entrance to the pore system, which can be exploited for separations. For example, the fcu-MOFs contain two types of cage (tetrahedron and octahedron) but only one type of window (triangle). By using 1,4naphthalenedicarboxylate as the organic linker, the bulky naphthalene reduces the window size, enabling separation of $n$-butane-methane, butanol-methanol and butanol-water pair systems driven by adsorption kinetics ${ }^{206}$.

Building blocks can also be substituted with other pre-designed building blocks, including another inorganic or organic building block, or even a group of building blocks with suitable net-coded geometry for desired applications. Simple examples include the replacement of one inorganic MBB by another, such as the replacement of hexanuclear $\mathrm{Zr}$ clusters with rare-earth clusters and vice versa ${ }^{58,60}$, or one organic MBB by another, such as the replacement of a 
carboxylate linker with an azolate linker ${ }^{58}$. The substitution of an inorganic MBB with an organic MBB and vice versa was demonstrated for the (4,12)-c shp and (6,12)-c alb platforms ${ }^{9}$. The initial shp-MOF and alb-MOF were designed using nonanuclear rare-earth clusters as 12c building units and organic linkers as 4-c or 6-c building units. The rare-earth clusters were subsequently replaced by 12-c organic linkers and the organic linkers replaced by 4-c $\mathrm{Cu}$ paddlewheel clusters or 6-c trinuclear In clusters, affording Cu-shp-MOF-1 and In-alb-MOF1 , respectively.

Post-synthesis design strategies have also been developed for the modification, exchange, insertion and polymerization of building blocks after MOF assembly ${ }^{205}$. Some post-synthesis strategies, such as post-synthetic modification and post-synthetic exchange, afford isoreticular structures, and are thus a practical approach for isoreticular design, whereas others, such as post-synthetic insertion and post-synthetic polymerization, afford structures with a different topology.

\section{[H2] The multivariate approach}

The MTV approach introduces multiple types of functional groups or metal atoms into one MOF. This approach was first demonstrated by introducing up to eight functional groups into the MOF-5 platform ${ }^{207}$, the distribution of which can be examined using solid-state nuclear magnetic resonance ${ }^{208}$. The MTV approach has subsequently been applied to other platforms, including the qom-MOF platform, whereby various functionalized BTB linkers were introduced into MOF-177, affording MTV-MOF-177 (ref. ${ }^{200}$ ). The linker in MTV-MOFs can be further modified. For example, the organic linker in MTV-MOF-74 was modified in a sevenstep reaction to covalently incorporate tripeptides that resemble the active sites of enzymes ${ }^{209}$. In another example, MTV-MOFs were synthesized with both thermostable and thermolabile linkers $^{210}$. The thermolabile linker can then be removed by high-temperature treatment, creating hierarchical pores.

Multi-metal MTV-MOFs have also been reported. For example, ten different metals were introduced into the rod building blocks of MOF-74, characterized by elemental analysis, inductively coupled plasma optical emission spectroscopies and energy-dispersive X-ray spectroscopies $^{211}$. Multiple metals were also introduced into the trinuclear clusters and porphyrin-based linkers of the stp-MOF platform, with the metals forming either domains or a well-mixed spatial arrangement ${ }^{39}$. 
Various routes have been explored to further control the position of building blocks in MTVMOFs. The concept of domain building blocks (DBBs) has recently been introduced for MTVMOFs containing distinct structural or compositional regions ${ }^{212}$. Using this concept, stratified MOF particles consisting of multiple concentric DBBs were synthesized through stepwise epitaxial growth. Moreover, in a method to control the relative position of linkers in MTVMOFs, imine-based interlocked ligands were mixed with the initial ligands in ZIF-8 $\left(\right.$ ref. $^{213}$ ). The imine fragments can then be removed by post-synthetic hydrolysis, leaving structures with controlled heterogeneity.

\section{[H2] Building block expansion and contraction}

The expansion and contraction of building blocks has, to date, mainly been based on the organic linkers, because the insertion of metal atoms to obtain larger clusters while maintaining the same geometry remains difficult.

The expansion of MBBs is an effective strategy to obtain materials with larger pore volumes. For example, the expansion of organic linkers in the MOF-74 family afforded an isoreticular series of MOF-74 structures with pore apertures of up to $98 \AA$ (ref. ${ }^{79}$ ). The expansion of rhtMOF-1 gave a series of large-pore rht-MOFs ${ }^{32,147}$, with one structure, NU-110, having a Brunauer-Emmett-Teller surface area of $\sim 7,000 \mathrm{~m}^{2} \mathrm{~g}^{-1}$ (ref. ${ }^{148}$ ). The expansion of the csqMOF platform, afforded a series of large-pore csq-MOFs with a pore-aperture diameter of up to $67 \AA$ in NU-1007 (ref. ${ }^{170}$ ).

Excess expansion of organic linkers can, however, decrease structural stability. The balance between larger pore volume and better material stability is an essential factor when targeting applications. One successful example is the expansion of the soc-MOF platform. The first socMOF comprised a trinuclear In cluster and a small linker, 3,3',5,5'-azobenzene tetracarboxylate $^{41}$. A MOF with much larger pore volume, Al-soc-MOF-1, can be obtained by using an extended ligand, 3,3",5,5"-tetrakis(4-carboxyphenyl)-p-terphenyl ${ }^{34}$. Al-soc-MOF-1 is the first MOF to fulfill the US Department of Energy's target for methane storage for both gravimetric and volumetric capacities. The $\mathrm{Cr}$ isomer, Cr-soc-MOF-1, owing to its high porosity and water stability, displays the record water vapour uptake of $1.95 \mathrm{~g} \mathrm{~g}^{-1}$ at $70 \%$ relative humidity ${ }^{35}$.

The contraction of building blocks can afford materials with reduced pore-aperture sizes, suitable for the separation of small molecules, and can sometimes give rise to more stable materials. In an example of linker contraction, the RE-fcu-MOF synthesized with fumaric acid 
separated branched paraffins from normal paraffins ${ }^{139}$. Post-synthesis expansion and contraction approaches have also been reported. For example, the ditopic linker in bio-MOF100 was exchanged in two steps, yielding bio-MOF-103 with an increased pore size of $2.9 \mathrm{~nm}$ $\left(\right.$ ref. $\left.^{180}\right)$. Recently, a sequential linker labilization and reinstallation method was reported as a more general post-synthesis approach for the expansion and contraction of $\mathrm{MOFs}^{214}$. A labile imine-based linker is introduced to mediate the exchange between linkers of different size, affording non-interpenetrated large-pore Zr-fcu-MOFs.

The expansion and contraction of materials based on merged nets are more challenging. A mathematical rule for the expansion and contraction of merged nets structures was introduced for sph-MOFs ${ }^{10}$. For merged nets, the expansion and contraction of structures need to meet a ratio constant, which is an intrinsic property of a merged net. Although a net is a mathematical concept and the edges of a net do not have specific lengths, in merged nets, the ratio between the edge lengths is fixed. The ratio constant of a merged net was defined as the ratio between length of the short and long edges, and will always be between 0 and 1 . For example, the ratio constant of the sph net is $\sqrt{3 / 3}(\sim 0.577)$. Thus, only when the edge length ratio between the spn net and hxg net is $\sqrt{3} / 3$ can these two nets merge to form the sph net (Fig. 9a). The sizes of organic linkers can then be pre-calculated and linkers of different sizes designed and paired before the synthesis of mixed-linker sph-MOFs (Fig. 9b).

\section{[H1] Outlook}

Many examples of synthesis by design attest that the design methodologies discussed above have become practical tools in periodic materials discovery. The ongoing advancement of these approaches points to a broad unknown space waiting for exploration. We believe that reticular chemistry will benefit from the exploration of additional directions for new materials discovery.

It is imperative to continue improving and enlarging the current reticular chemistry design toolbox. The number of MBBs, SBLs and SBBs that can be reliably obtained is still limited and insufficient to provide the desired net-cBUs for the reticulation of many nets in the RCSR database. It is especially urgent to develop highly connected clusters to fulfill 12-c building units (truncated tetrahedra and icosahedra) and 24-c building units (rhombicuboctahedra and truncated octahedra), which would provide access to highly coordinated platforms such as ttt, mgc, ith, rht and twf. The importance of these approaches will continue to increase: although many simple structures can be obtained directly by trial and error, the size, angle and other 
geometry-coded information of more intricate structures are constrained and must be predesigned to fulfill all the requirements.

Until now, the use of these design tools has mostly been disjointed. A rational combination of different design strategies, such as the SBB approach, merged-net approach and MTV approach, could lead to platforms with unprecedented control of functional groups in materials. The development of 24-c, or even 36-c SBBs, would provide numerous new opportunities for the practice of the merged-net approach.

Examples of structures based on merged nets are still very limited, and many possibilities of intricate designable platforms are awaiting discovery. Deeper understanding of the underlying principles and the systematic enumeration of merged nets will be important for the design of more intricate structures in reticular chemistry. Although the merged-net approach has only been applied to MOFs to date, it could also be applicable to COFs. In theory, the merging concept is not limited to inorganic MBBs, as it could be practiced using multiple branched organic MBBs. An organic linker with branched coordinating functionalities could also connect to distinct inorganic MBBs.

Compared with three-periodic MOFs, there are fewer examples of three-periodic $\mathrm{COFs}^{215}$, as they are difficult to crystallize. The first single-crystal X-ray diffraction structures of COFs were reported in 2018 (ref. $^{216}$ ). The exploration of rigid, highly connected organic linkers with proper shapes, such as cuboctahedron and hexagonal prism, is also crucial for the construction of three-periodic COFs. As COFs are based on two-periodic layers, the SBL approach warrants further attention in the search for three-periodic COFs.

An interesting question is whether we can rationally design materials with ordered combinations of many geometrically variant metal clusters and organic linkers in one structure. In such a material, each type of building block would embed distinct functional groups that could cooperate with each other to undertake convoluted applications.

\section{References}

1. Ockwig, N. W., Delgado-Friedrichs, O., O'Keeffe, M. \& Yaghi, O. M. Reticular Chemistry: Occurrence and Taxonomy of Nets and Grammar for the Design of Frameworks. Acc. Chem. Res. 38, 176-182, (2005).

2. Yaghi, O. M. Reticular Chemistry-Construction, Properties, and Precision Reactions of Frameworks. J. Am. Chem. Soc. 138, 15507-15509 (2016).

3. Jia, J. T. et al. Extremely Hydrophobic POPs to Access Highly Porous Storage Media and Capturing Agent for Organic Vapors. Chem 5, 180-191 (2019).

4. Diercks, C. S. \& Yaghi, O. M. The atom, the molecule, and the covalent organic framework. Science 355, eaal1585 (2017). 
5. Yaghi, O. M., Kalmutzki, M. J. \& Diercks, C. S. Introduction to Reticular Chemistry: Metal-Organic Frameworks and Covalent Organic Frameworks. (John Wiley \& Sons, 2019).

6. Yaghi, O. M. Reticular Chemistry in All Dimensions. ACS Central Science 5, 1295-1300 (2019).

7. O'Keeffe, M., Peskov, M. A., Ramsden, S. J. \& Yaghi, O. M. The Reticular Chemistry Structure Resource (RCSR) Database of, and Symbols for, Crystal Nets. Acc. Chem. Res. 41, 1782-1789 (2008).

8. Chen, Z., Jiang, H., O'Keeffe, M. \& Eddaoudi, M. Minimal edge-transitive nets for the design and construction of metal-organic frameworks. Faraday Discuss. 201, 127-143 (2017).

9. Chen, Z. et al. Applying the Power of Reticular Chemistry to Finding the Missing alb-MOF Platform Based on the (6,12)-Coordinated Edge-Transitive Net. J. Am. Chem. Soc. 139, 3265-327 (2017).

10. Jiang, H. et al. Enriching the Reticular Chemistry Repertoire: Merged Nets Approach for the Rational Design of Intricate Mixed-Linker Metal-Organic Framework Platforms. J. Am. Chem. Soc. 140, 8858-8867 (2018).

11. Eddaoudi, M., Sava, D. F., Eubank, J. F., Adil, K. \& Guillerm, V. Zeolite-like metal-organic frameworks (ZMOFs): design, synthesis, and properties. Chem. Soc. Rev. 44, 228-249 (2015).

12. Banerjee, R. et al. High-Throughput Synthesis of Zeolitic Imidazolate Frameworks and Application to $\mathrm{CO}_{2}$ Capture. Science 319, 939-943 (2008).

13.Yang, J. et al. Principles of Designing Extra-Large Pore Openings and Cages in Zeolitic Imidazolate Frameworks. J. Am. Chem. Soc. 139, 6448-6455 (2017).

14. Liu, Y., Kravtsov, V. C., Larsen, R. \& Eddaoudi, M. Molecular building blocks approach to the assembly of zeolite-like metal-organic frameworks (ZMOFs) with extra-large cavities. Chem. Commun., 1488-1490 (2006).

15. Liu, Y., Kravtsov, V. C. \& Eddaoudi, M. Template-Directed Assembly of Zeolite-like Metal-Organic Frameworks (ZMOFs): a usf-ZMOF with an Unprecedented Zeolite Topology. Angew. Chem. Int. Ed. 47, 8446-8449 (2008).

16. Wu, T. et al. Zeolite RHO-Type Net with the Lightest Elements. J. Am. Chem. Soc. 131, 6111-6113 (2009).

17. Zhang, J. et al. Zeolitic Boron Imidazolate Frameworks. Angew. Chem. Int. Ed. 48, 2542-2545 (2009).

18. Cadiau, A. et al. Hydrolytically stable fluorinated metal-organic frameworks for energy-efficient dehydration. Science 356, 731-735, (2017).

19. Belmabkhout, Y. et al. Natural gas upgrading using a fluorinated $\mathrm{MOF}$ with tuned $\mathrm{H}_{2} \mathrm{~S}$ and $\mathrm{CO}_{2}$ adsorption selectivity. Nature Energy 3, 1059-1066 (2018).

20. Tchalala, M. R. et al. Fluorinated MOF platform for selective removal and sensing of $\mathrm{SO}_{2}$ from flue gas and air. Nature Communications 10, 1328 (2019).

21. Nugent, P. et al. Porous materials with optimal adsorption thermodynamics and kinetics for $\mathrm{CO}_{2}$ separation. Nature 495, 80-84 (2013).

22. Subramanian, S. \& Zaworotko, M. J. Porous Solids by Design: $\left[\mathrm{Zn}\left(4,4^{\prime}-\text {-bpy }\right)_{2}\left(\mathrm{SiF}_{6}\right)\right] n \cdot x \mathrm{DMF}$, a Single Framework Octahedral Coordination Polymer with Large Square Channels. Angew. Chem. Int. Ed. Engl. 34, 2127-2129 (1995).

23. Shekhah, O. et al. A facile solvent-free synthesis route for the assembly of a highly $\mathrm{CO}_{2}$ selective and $\mathrm{H}_{2} \mathrm{~S}$ tolerant NiSIFSIX metal-organic framework. Chem. Commun. 51, 13595-13598 (2015).

24.Shekhah, O. et al. Made-to-order metal-organic frameworks for trace carbon dioxide removal and air capture. Nature Communications 5, 4228 (2014).

25. Bhatt, P. M. et al. A Fine-Tuned Fluorinated MOF Addresses the Needs for Trace $\mathrm{CO}_{2}$ Removal and Air Capture Using Physisorption. J. Am. Chem. Soc. 138, 9301-9307 (2016).

26. Cadiau, A., Adil, K., Bhatt, P. M., Belmabkhout, Y. \& Eddaoudi, M. A metal-organic framework-based splitter for separating propylene from propane. Science 353, 137-140 (2016).

27. Adil, K. et al. Valuing Metal-Organic Frameworks for Postcombustion Carbon Capture: a Benchmark Study for Evaluating Physical Adsorbents. Adv. Mater. 29, 1702953 (2017).

28. Chui, S. S.-Y., Lo, S. M.-F., Charmant, J. P. H., Orpen, A. G. \& Williams, I. D. A Chemically Functionalizable Nanoporous Material $\left[\mathrm{Cu}_{3}(\mathrm{TMA})_{2}\left(\mathrm{H}_{2} \mathrm{O}\right)_{3}\right]_{n}$. Science 283, 1148-1150 (1999).

29. Eubank, J. F. et al. The Next Chapter in MOF Pillaring Strategies: Trigonal Heterofunctional Ligands To Access Targeted High-Connected Three Dimensional Nets, Isoreticular Platforms. J. Am. Chem. Soc. 133, 1753217535 (2011).

30. Chun, H. Low-Level Self-Assembly of Open Framework Based on Three Different Polyhedra: Metal-Organic Analogue of Face-Centered Cubic Dodecaboride. J. Am. Chem. Soc. 130, 800-801 (2008).

31. Tu, B. et al. Reversible Redox Activity in Multicomponent Metal-Organic Frameworks Constructed from Trinuclear Copper Pyrazolate Building Blocks. J. Am. Chem. Soc. 139, 7998-8007 (2017).

32. Nouar, F. et al. Supermolecular Building Blocks (SBBs) for the Design and Synthesis of Highly Porous MetalOrganic Frameworks. J. Am. Chem. Soc. 130, 1833-1835 (2008).

33. Quartapelle Procopio, E. et al. Cation-Exchange Porosity Tuning in Anionic Metal-Organic Frameworks for the Selective Separation of Gases and Vapors and for Catalysis. Angew. Chem. Int. Ed. 49, 7308-7311 (2010). 
34. Alezi, D. et al. MOF Crystal Chemistry Paving the Way to Gas Storage Needs: aluminum-Based soc-MOF for $\mathrm{CH}_{4}, \mathrm{O}_{2}$, and $\mathrm{CO}_{2}$ Storage. J. Am. Chem. Soc. 137, 13308-13318 (2015).

35. Towsif Abtab, S. M. et al. Reticular Chemistry in Action: A Hydrolytically Stable MOF Capturing Twice Its Weight in Adsorbed Water. Chem 4, 94-105 (2018).

36. Serre, C., Millange, F., Surblé, S. \& Férey, G. A Route to the Synthesis of Trivalent Transition-Metal Porous Carboxylates with Trimeric Secondary Building Units. Angew. Chem. Int. Ed. 43, 6285-6289 (2004).

37. Férey, G. et al. A Chromium Terephthalate-Based Solid with Unusually Large Pore Volumes and Surface Area. Science 309, 2040-2042(2005).

38. Feng, D. et al. Stable metal-organic frameworks containing single-molecule traps for enzyme encapsulation. Nature Communications 6, 5979 (2015).

39. Liu, Q., Cong, H. \& Deng, H. Deciphering the Spatial Arrangement of Metals and Correlation to Reactivity in Multivariate Metal-Organic Frameworks. J. Am. Chem. Soc. 138, 13822-13825 (2016).

40. Jia, J. et al. Highly porous and robust ionic MOFs with nia topology constructed by connecting an octahedral ligand and a trigonal prismatic metal cluster. Chem. Commun. 48, 6010-6012 (2012).

41. Liu, Y. et al. Assembly of Metal-Organic Frameworks (MOFs) Based on Indium-Trimer Building Blocks: a Porous MOF with soc Topology and High Hydrogen Storage. Angew. Chem. Int. Ed. 46, 3278-3283 (2007).

42. Zhang, Y.-B., Zhang, W.-X., Feng, F.-Y., Zhang, J.-P. \& Chen, X.-M. A Highly Connected Porous Coordination Polymer with Unusual Channel Structure and Sorption Properties. Angew. Chem. Int. Ed. 48, 5287-5290 (2009).

43. Jiang, G. et al. A Nine-Connected Mixed-Ligand Nickel-Organic Framework and Its Gas Sorption Properties. Crystal Growth \& Design 11, 3713-3716 (2011).

44. Zhang, Y.-B. et al. Geometry analysis and systematic synthesis of highly porous isoreticular frameworks with a unique topology. Nature Communications 3, 642 (2012).

45. Zhao, X. et al. Selective anion exchange with nanogated isoreticular positive metal-organic frameworks. Nature Communications 4, 2344 (2013).

46. Zhai, Q.-G. et al. An ultra-tunable platform for molecular engineering of high-performance crystalline porous materials. Nature Communications 7, 13645 (2016).

47. Li, H., Eddaoudi, M., O'Keeffe, M. \& Yaghi, O. M. Design and synthesis of an exceptionally stable and highly porous metal-organic framework. Nature 402, 276-279 (1999).

48. Eddaoudi, M. et al. Systematic Design of Pore Size and Functionality in Isoreticular MOFs and Their Application in Methane Storage. Science 295, 469-472 (2002).

49. Montoro, C. et al. Capture of Nerve Agents and Mustard Gas Analogues by Hydrophobic Robust MOF-5 Type Metal-Organic Frameworks. J. Am. Chem. Soc. 133, 11888-11891 (2011).

50. Brozek, C. K. \& Dincă, M. $\mathrm{Ti}^{3+}-, \mathrm{V}^{2+/ 3+}-, \mathrm{Cr}^{2+/ 3+}-, \mathrm{Mn}^{2+}-$, and $\mathrm{Fe}^{2+}-$ Substituted MOF-5 and Redox Reactivity in Crand Fe-MOF-5. J. Am. Chem. Soc. 135, 12886-12891, (2013).

51. Luo, T.-Y. et al. Rare Earth pcu Metal-Organic Framework Platform Based on $\mathrm{RE}_{4}\left(\mu_{3}-\mathrm{OH}\right)_{4}(\mathrm{COO})_{6}{ }^{2+}$ Clusters: Rational Design, Directed Synthesis, and Deliberate Tuning of Excitation Wavelengths. J. Am. Chem. Soc. 139, 9333-9340 (2017).

52. Chen, D.-m., Shi, W. \& Cheng, P. A cage-based cationic body-centered tetragonal metal-organic framework: single-crystal to single-crystal transformation and selective uptake of organic dyes. Chem. Commun. 51, 370372 (2015).

53. Wang, D., Zhao, T., Li, G., Huo, Q. \& Liu, Y. A porous sodalite-type MOF based on tetrazolcarboxylate ligands and $\left[\mathrm{Cu}_{4} \mathrm{Cl}\right]^{7+}$ squares with open metal sites for gas sorption. Dalton Transactions 43, 2365-2368 (2014).

54. Dincă, M. et al. Hydrogen Storage in a Microporous Metal-Organic Framework with Exposed $\mathrm{Mn}^{2+}$ Coordination Sites. J. Am. Chem. Soc. 128, 16876-16883 (2006).

55. Castells-Gil, J. et al. Chemical Engineering of Photoactivity in Heterometallic Titanium-Organic Frameworks by Metal Doping. Angew. Chem. Int. Ed. 57, 8453-8457 (2018).

56. Lammert, M. et al. Cerium-based metal organic frameworks with UiO-66 architecture: synthesis, properties and redox catalytic activity. Chem. Commun. 51, 12578-12581 (2015).

57. Cavka, J. H. et al. A New Zirconium Inorganic Building Brick Forming Metal Organic Frameworks with Exceptional Stability. J. Am. Chem. Soc. 130, 13850-13851 (2008).

58. Xue, D.-X. et al. Tunable Rare-Earth fcu-MOFs: a Platform for Systematic Enhancement of $\mathrm{CO}_{2} \mathrm{Adsorption}$ Energetics and Uptake. J. Am. Chem. Soc. 135, 7660-7667, (2013).

59. Xue, D.-X. et al. Topology meets MOF chemistry for pore-aperture fine tuning: ftw-MOF platform for energyefficient separations via adsorption kinetics or molecular sieving. Chem. Commun. 54, 6404-6407 (2018).

60. Furukawa, H. et al. Water Adsorption in Porous Metal-Organic Frameworks and Related Materials. J. Am. Chem. Soc. 136, 4369-4381 (2014). 
61. Pang, J. et al. Control the Structure of Zr-Tetracarboxylate Frameworks through Steric Tuning. J. Am. Chem. Soc. 139, 16939-16945 (2017).

62. Alezi, D. et al. Quest for Highly Connected Metal-Organic Framework Platforms: rare-Earth Polynuclear Clusters Versatility Meets Net Topology Needs. J. Am. Chem. Soc. 137, 5421-5430 (2015).

63. Alezi, D. et al. Reticular Chemistry at Its Best: directed Assembly of Hexagonal Building Units into the Awaited Metal-Organic Framework with the Intricate Polybenzene Topology, pbz-MOF. J. Am. Chem. Soc. 138, 1276712770 (2016).

64. Feng, D. et al. A Highly Stable Zeotype Mesoporous Zirconium Metal-Organic Framework with Ultralarge Pores. Angew. Chem. Int. Ed. 54, 149-154 (2015).

65. Liu, Q. et al. Mesoporous Cages in Chemically Robust MOFs Created by a Large Number of Vertices with Reduced Connectivity. J. Am. Chem. Soc. 141, 488-496 (2019).

66. Wang, H. et al. Topologically guided tuning of Zr-MOF pore structures for highly selective separation of $\mathrm{C6}$ alkane isomers. Nature Communications 9, 1745 (2018).

67. Zhang, Y. et al. A Flexible Metal-Organic Framework with 4-Connected Zr6 Nodes. J. Am. Chem. Soc. 140, 11179-11183 (2018).

68. Masciocchi, N. et al. Cubic Octanuclear Ni(II) Clusters in Highly Porous Polypyrazolyl-Based Materials. J. Am. Chem. Soc. 132, 7902-7904 (2010).

69. Holmberg, R. J. et al. An unprecedented Co"l cuboctahedron as the secondary building unit in a Co-based metal-organic framework. Chem. Commun. 50, 5333-5335 (2014).

70. Feng, D. et al. Metal-Organic Frameworks Based on Previously Unknown Zr $8 / \mathrm{Hf}_{8}$ Cubic Clusters. Inorg. Chem. 52, 12661-12667 (2013).

71. Luebke, R. et al. Versatile rare earth hexanuclear clusters for the design and synthesis of highly-connected ftw-MOFs. Chem. Sci. 6, 4095-4102 (2015).

72. Kapustin, E. A., Lee, S., Alshammari, A. S. \& Yaghi, O. M. Molecular Retrofitting Adapts a Metal-Organic Framework to Extreme Pressure. ACS Central Science 3, 662-667 (2017).

73. Gándara, F., Furukawa, H., Lee, S. \& Yaghi, O. M. High Methane Storage Capacity in Aluminum Metal-Organic Frameworks. J. Am. Chem. Soc. 136, 5271-5274 (2014).

74.Lee, S., Kapustin, E. A. \& Yaghi, O. M. Coordinative alignment of molecules in chiral metal-organic frameworks. Science 353, 808-811 (2016).

75. Ahnfeldt, T. et al. $\left[\mathrm{Al} \mathrm{I}_{4}(\mathrm{OH})_{2}\left(\mathrm{OCH}_{3}\right)_{4}\left(\mathrm{H}_{2} \mathrm{~N}-\mathrm{bdc}\right)_{3}\right] \cdot x \mathrm{H}_{2} \mathrm{O}$ : a 12-Connected Porous Metal-Organic Framework with an Unprecedented Aluminum-Containing Brick. Angew. Chem. Int. Ed. 48, 5163-5166 (2009).

76. Dan-Hardi, M. et al. A New Photoactive Crystalline Highly Porous Titanium(IV) Dicarboxylate. J. Am. Chem. Soc. 131, 10857-10859 (2009).

77. Guillerm, V. et al. Discovery and introduction of a $(3,18)$-connected net as an ideal blueprint for the design of metal-organic frameworks. Nature Chemistry 6, 673-680 (2014).

78. Schoedel, A., Li, M., Li, D., O’Keeffe, M. \& Yaghi, O. M. Structures of Metal-Organic Frameworks with Rod Secondary Building Units. Chem. Rev. 116, 12466-12535 (2016).

79.Deng, H. et al. Large-Pore Apertures in a Series of Metal-Organic Frameworks. Science 336, 1018-1023 (2012).

80. Kapelewski, M. T. et al. $\mathrm{M} 2$ (m-dobdc) ( $\mathrm{M}=\mathrm{Mg}, \mathrm{Mn}$, Fe, Co, Ni) Metal-Organic Frameworks Exhibiting Increased Charge Density and Enhanced H2 Binding at the Open Metal Sites. J. Am. Chem. Soc. 136, 1211912129, (2014).

81. McDonald, T. M. et al. Capture of Carbon Dioxide from Air and Flue Gas in the Alkylamine-Appended MetalOrganic Framework mmen-Mg2 (dobpdc). J. Am. Chem. Soc. 134, 7056-7065 (2012).

82. Groves, J. A. et al. The first route to large pore metal phosphonates. Chem. Commun., 3305-3307 (2006).

83. Miller, S. R. et al. Structural Transformations and Adsorption of Fuel-Related Gases of a Structurally Responsive Nickel Phosphonate Metal-Organic Framework, Ni-STA-12. J. Am. Chem. Soc. 130, 15967-15981 (2008).

84. Wharmby, M. T., Mowat, J. P. S., Thompson, S. P. \& Wright, P. A. Extending the Pore Size of Crystalline Metal Phosphonates toward the Mesoporous Regime by Isoreticular Synthesis. J. Am. Chem. Soc. 133, 1266-1269 (2011).

85. Catarineu, N. R. et al. Two Principles of Reticular Chemistry Uncovered in a Metal-Organic Framework of Heterotritopic Linkers and Infinite Secondary Building Units. J. Am. Chem. Soc. 138, 10826-10829 (2016).

86. Herm, Z. R. et al. Separation of Hexane Isomers in a Metal-Organic Framework with Triangular Channels. Science 340, 960-964 (2013).

87. Yang, S. et al. Selectivity and direct visualization of carbon dioxide and sulfur dioxide in a decorated porous host. Nature Chemistry 4, 887-894 (2012). 
88. Dai, H. et al. Reticulation of 2D Semiconductors by Metal-Organic Approach for Efficient Hydrogen Evolution. ACS Sustainable Chemistry \& Engineering 8, 8102-8110 (2020).

89. Eddaoudi, M. et al. Porous Metal-Organic Polyhedra: $25 \AA ̊$ Cuboctahedron Constructed from $12 \mathrm{Cu}_{2}\left(\mathrm{CO}_{2}\right)_{4}$ Paddle-Wheel Building Blocks. J. Am. Chem. Soc. 123, 4368-4369 (2001).

90. Fujita, D. et al. Self-assembly of tetravalent Goldberg polyhedra from 144 small components. Nature 540, 563-566 (2016).

91. Sun, Q.-F. et al. Self-Assembled $\mathrm{M}_{24} \mathrm{~L}_{48}$ Polyhedra and Their Sharp Structural Switch upon Subtle Ligand Variation. Science 328, 1144-1147 (2010).

92. Guillerm, V. et al. A supermolecular building approach for the design and construction of metal-organic frameworks. Chem. Soc. Rev. 43, 6141-6172 (2014).

93. Bon, V., Senkovskyy, V., Senkovska, I. \& Kaskel, S. Zr(IV) and Hf(IV) based metal-organic frameworks with reotopology. Chem. Commun. 48, 8407-8409 (2012).

94. Sun, X. et al. A Stable Mesoporous Zr-Based Metal Organic Framework for Highly Efficient $\mathrm{CO}_{2} \mathrm{Conversion.}$ Inorg. Chem. 58, 7480-7487 (2019).

95. Xie, L.-H., Liu, X.-M., He, T. \& Li, J.-R. Metal-Organic Frameworks for the Capture of Trace Aromatic Volatile Organic Compounds. Chem 4, 1911-1927 (2018).

96. Park, I.-H., Lee, E., Lee, S. S. \& Vittal, J. J. Chemical Patterning in Single Crystals of Metal-Organic Frameworks by [2+2] Cycloaddition Reaction. Angew. Chem. Int. Ed. 58, 14860-14864 (2019).

97. He, Y., Li, B., O'Keeffe, M. \& Chen, B. Multifunctional metal-organic frameworks constructed from metabenzenedicarboxylate units. Chem. Soc. Rev. 43, 5618-5656 (2014).

98. Eddaoudi, M., Kim, J., O'Keeffe, M. \& Yaghi, O. M. $\mathrm{Cu}_{2}\left[\mathrm{O}-\mathrm{Br}-\mathrm{C}_{6} \mathrm{H}_{3}\left(\mathrm{CO}_{2}\right)_{2}\right]_{2}\left(\mathrm{H}_{2} \mathrm{O}\right)_{2} \cdot(\mathrm{DMF})_{8}\left(\mathrm{H}_{2} \mathrm{O}\right)_{2}$ : a Framework Deliberately Designed To Have the NbO Structure Type. J. Am. Chem. Soc. 124, 376-377 (2002).

99. Li, P. et al. Bottom-up construction of a superstructure in a porous uranium-organic crystal. Science 356, 624627 (2017).

100. Guillerm, V. \& Maspoch, D. Geometry Mismatch and Reticular Chemistry: Strategies To Assemble MetalOrganic Frameworks with Non-default Topologies. J. Am. Chem. Soc. 141, 16517-16538 (2019).

101. Guillerm, V., Grancha, T., Imaz, I., Juanhuix, J. \& Maspoch, D. Zigzag Ligands for Transversal Design in Reticular Chemistry: Unveiling New Structural Opportunities for Metal-Organic Frameworks. J. Am. Chem. Soc. 140, 10153-10157 (2018).

102. Nguyen, T. T. M., Le, H. M., Kawazoe, Y. \& Nguyen, H. L. Reticular control of interpenetration in a complex metal-organic framework. Materials Chemistry Frontiers 2, 2063-2069 (2018).

103. Benecke, J. et al. Polymorphous Indium Metal-Organic Frameworks Based on a Ferrocene Linker: Redox Activity, Porosity, and Structural Diversity. Inorg. Chem. 59, 9969-9978 (2020).

104. Ni, Z., Yassar, A., Antoun, T. \& Yaghi, O. M. Porous Metal-Organic Truncated Octahedron Constructed from Paddle-Wheel Squares and Terthiophene Links. J. Am. Chem. Soc. 127, 12752-12753 (2005).

105. Li, J.-R., Timmons, D. J. \& Zhou, H.-C. Interconversion between Molecular Polyhedra and Metal-Organic Frameworks. J. Am. Chem. Soc. 131, 6368-6369 (2009).

106. Li, J.-R. et al. Ligand Bridging-Angle-Driven Assembly of Molecular Architectures Based on Quadruply Bonded Mo-Mo Dimers. J. Am. Chem. Soc. 132, 17599-17610 (2010).

107. Gosselin, E. J., Rowland, C. A., Balto, K. P., Yap, G. P. A. \& Bloch, E. D. Design and Synthesis of Porous Nickel(II) and Cobalt(II) Cages. Inorg. Chem. 57, 11847-11850 (2018).

108. Rowland, C. A. et al. Methane Storage in Paddlewheel-Based Porous Coordination Cages. J. Am. Chem. Soc. 140, 11153-11157 (2018).

109. Cairns, A. J. et al. Supermolecular Building Blocks (SBBs) and Crystal Design: 12-Connected Open Frameworks Based on a Molecular Cubohemioctahedron. J. Am. Chem. Soc. 130, 1560-1561 (2008).

110. Stoeck, U., Krause, S., Bon, V., Senkovska, I. \& Kaskel, S. A highly porous metal-organic framework, constructed from a cuboctahedral super-molecular building block, with exceptionally high methane uptake. Chem. Commun. 48, 10841-10843 (2012).

111. Krause, S. et al. A pressure-amplifying framework material with negative gas adsorption transitions. Nature 532, 348-352 (2016).

112. Stoeck, U., Senkovska, I., Bon, V., Krause, S. \& Kaskel, S. Assembly of metal-organic polyhedra into highly porous frameworks for ethene delivery. Chem. Commun. 51, 1046-1049 (2015).

113. Zou, Y., Park, M., Hong, S. \& Lah, M. S. A designed metal-organic framework based on a metal-organic polyhedron. Chem. Commun., 2340-2342 (2008).

114. Zhang, P., Li, B., Zhao, Y., Meng, X. \& Zhang, T. A novel $(3,36)$-connected and self-interpenetrated metalorganic framework with high thermal stability and gas-sorption capabilities. Chem. Commun. 47, 7722-7724 (2011). 
115. Lu, Z. et al. The Utilization of Amide Groups To Expand and Functionalize Metal-Organic Frameworks Simultaneously. Chemistry - A European Journal 22, 6277-6285 (2016).

116. Li, J.-R. \& Zhou, H.-C. Metal-Organic Hendecahedra Assembled from Dinuclear Paddlewheel Nodes and Mixtures of Ditopic Linkers with 120 and 90 Bend Angles. Angew. Chem. Int. Ed. 48, 8465-8468 (2009).

117. Slater, A. G. et al. Reticular synthesis of porous molecular 1D nanotubes and 3D networks. Nature Chemistry 9, 17-25 (2016).

118. Eubank, J. F. et al. The Quest for Modular Nanocages: tbo-MOF as an Archetype for Mutual Substitution, Functionalization, and Expansion of Quadrangular Pillar Building Blocks. J. Am. Chem. Soc. 133, 14204-14207 (2011).

119. Chun, H., Dybtsev, D. N., Kim, H. \& Kim, K. Synthesis, X-ray Crystal Structures, and Gas Sorption Properties of Pillared Square Grid Nets Based on Paddle-Wheel Motifs: Implications for Hydrogen Storage in Porous Materials. 11, 3521-3529 (2005).

120. Ma, B.-Q., Mulfort, K. L. \& Hupp, J. T. Microporous Pillared Paddle-Wheel Frameworks Based on MixedLigand Coordination of Zinc lons. Inorg. Chem. 44, 4912-4914 (2005).

121. Seki, K., Takamizawa, S. \& Mori, W. Design and Gas Adsorption Property of a Three-Dimensional Coordination Polymer with a Stable and Highly Porous Framwork. Chem. Lett. 30, 332-333 (2001).

122. Spanopoulos, I. et al. Reticular Synthesis of HKUST-like tbo-MOFs with Enhanced $\mathrm{CH}_{4}$ Storage. J. Am. Chem. Soc. 138, 1568-1574 (2016).

123. Moreau, F. et al. Tailoring porosity and rotational dynamics in a series of octacarboxylate metal-organic frameworks. Proc. Natl Acad. Sci. USA 114, 3056-3061 (2017).

124. Liu, X., Oh, M. \& Lah, M. S. Size- and Shape-Selective Isostructural Microporous Metal-Organic Frameworks with Different Effective Aperture Sizes. Inorg. Chem. 50, 5044-5053 (2011).

125. Chen, Z., Adil, K., Weseliński, Ł. J., Belmabkhout, Y. \& Eddaoudi, M. A supermolecular building layer approach for gas separation and storage applications: the eea and rtl MOF platforms for $\mathrm{CO}_{2}$ capture and hydrocarbon separation. Journal of Materials Chemistry A 3, 6276-6281 (2015).

126. Wang, R. et al. Porous Zirconium Metal - Organic Framework Constructed from 2D $\rightarrow$ 3D Interpenetration Based on a 3,6-Connected kgd Net. Inorg. Chem. 53, 7086-7088 (2014).

127. Li, M., O'Keeffe, M., Proserpio, D. M. \& Zhang, H.-F. A New Group of Edge-transitive 3-Periodic Nets and Their Derived Nets for Reticular Chemistry. Crystal Growth \& Design 20, 4062-4068 (2020).

128. Chen, Z., Jiang, H., Li, M., O’Keeffe, M. \& Eddaoudi, M. Reticular Chemistry 3.2: Typical Minimal EdgeTransitive Derived and Related Nets for the Design and Synthesis of Metal-Organic Frameworks. Chem. Rev. 120, 8039-8065 (2020).

129. Li, M., Li, D., O'Keeffe, M. \& Yaghi, O. M. Topological Analysis of Metal-Organic Frameworks with Polytopic Linkers and/or Multiple Building Units and the Minimal Transitivity Principle. Chem. Rev. 114, 13431370 (2014).

130. Chen, Z. et al. Enriching the Reticular Chemistry Repertoire with Minimal Edge-Transitive Related Nets: Access to Highly Coordinated Metal-Organic Frameworks Based on Double Six-Membered Rings as NetCoded Building Units. J. Am. Chem. Soc. 141, 20480-20489 (2019).

131. Feng, D. et al. Construction of Ultrastable Porphyrin Zr Metal-Organic Frameworks through Linker Elimination. J. Am. Chem. Soc. 135, 17105-17110 (2013).

132. Gomez-Gualdron, D. A. et al. Evaluating topologically diverse metal-organic frameworks for cryoadsorbed hydrogen storage. Energy Environ. Sci. 9, 3279-3289 (2016).

133. Nguyen, P. T. K. et al. New Metal-Organic Frameworks for Chemical Fixation of CO2. ACS Applied Materials \& Interfaces 10, 733-744 (2018).

134. He, Y. et al. Low-energy regeneration and high productivity in a lanthanide-hexacarboxylate framework for high-pressure $\mathrm{CO}_{2}-\mathrm{CH}_{4}-\mathrm{H}_{2}$ separation. Chem. Commun. 49, 6773-6775 (2013).

135. Wang, B. et al. Highly Stable Zr(IV)-Based Metal-Organic Frameworks for the Detection and Removal of Antibiotics and Organic Explosives in Water. J. Am. Chem. Soc. 138, 6204-6216 (2016).

136. Liu, T.-F. et al. Adding to the Arsenal of Zirconium-Based Metal-Organic Frameworks: the Topology as a Platform for Solvent-Assisted Metal Incorporation. Eur. J. Inorg. Chem. 2016, 4349-4352 (2016).

137. Zhang, M. et al. Symmetry-Guided Synthesis of Highly Porous Metal-Organic Frameworks with Fluorite Topology. Angew. Chem. Int. Ed. 53, 815-818 (2014).

138. Liu, B., Wu, W.-P., Hou, L., Li, Z.-S. \& Wang, Y.-Y. Two Nanocage-Based Metal-Organic Frameworks with Mixed-Cluster SBUs and $\mathrm{CO}_{2}$ Sorption Selectivity. Inorg. Chem. 54, 8937-8942 (2015).

139. Assen, A. H. et al. Ultra-Tuning of the Rare-Earth fcu-MOF Aperture Size for Selective Molecular Exclusion of Branched Paraffins. Angew. Chem. Int. Ed. 54, 14353-14358 (2015). 
140. Morris, W. et al. Synthesis, Structure, and Metalation of Two New Highly Porous Zirconium MetalOrganic Frameworks. Inorg. Chem. 51, 6443-6445 (2012).

141. Liu, T. F. et al. Topology-guided design and syntheses of highly stable mesoporous porphyrinic zirconium metal-organic frameworks with high surface area. J. Am. Chem. Soc. 137, 413-419 (2015).

142. Zhang, Q. et al. Förster Energy Transport in Metal-Organic Frameworks Is Beyond Step-by-Step Hopping. J. Am. Chem. Soc. 138, 5308-5315 (2016).

143. Feng, D. et al. A Highly Stable Porphyrinic Zirconium Metal-Organic Framework with shp-a Topology. J. Am. Chem. Soc. 136, 17714-17717, (2014).

144. AbdulHalim, R. G. et al. A Fine-Tuned Metal-Organic Framework for Autonomous Indoor Moisture Control. J. Am. Chem. Soc. 139, 10715-10722 (2017).

145. Gilson, S. E. et al. In Situ Formation of Unprecedented Neptunium-Oxide Wheel Clusters Stabilized in a Metal-Organic Framework. J. Am. Chem. Soc. 141, 11842-11846 (2019).

146. Chen, Z. et al. Ligand-Directed Reticular Synthesis of Catalytically Active Missing Zirconium-Based MetalOrganic Frameworks. J. Am. Chem. Soc. 141, 12229-12235 (2019).

147. Eubank, J. F. et al. On Demand: The Singular rht Net, an Ideal Blueprint for the Construction of a MetalOrganic Framework (MOF) Platform. Angew. Chem. Int. Ed. 51, 10099-10103 (2012).

148. Farha, O. K. et al. Metal-Organic Framework Materials with Ultrahigh Surface Areas: Is the Sky the Limit? J. Am. Chem. Soc. 134, 15016-15021 (2012).

149. Sun, Q. et al. A novel polyhedron-based metal-organic framework with high performance for gas uptake and light hydrocarbon separation. Dalton Transactions 47, 5005-5010 (2018).

150. Tu, B. et al. Ordered Vacancies and Their Chemistry in Metal-Organic Frameworks. J. Am. Chem. Soc. 136, 14465-14471 (2014).

151. Wu, H., Yang, J., Su, Z.-M., Batten, S. R. \& Ma, J.-F. An Exceptional 54-Fold Interpenetrated Coordination Polymer with 103-srs Network Topology. J. Am. Chem. Soc. 133, 11406-11409 (2011).

152. Furukawa, H. et al. Isoreticular Expansion of Metal-Organic Frameworks with Triangular and Square Building Units and the Lowest Calculated Density for Porous Crystals. Inorg. Chem. 50, 9147-9152 (2011).

153. Abrahams, B. F., Batten, S. R., Hamit, H., Hoskins, B. F. \& Robson, R. A Cubic $(3,4)$-Connected Net with Large Cavities in Solvated $\left[\mathrm{Cu}_{3}(\mathrm{tpt})_{4}\right]\left(\mathrm{ClO}_{4}\right)_{3}$ (tpt = 2,4,6-tri(4-pyridyl)-1,3,5-triazine). Angew. Chem. Int. Ed. Engl. 35, 1690-1692 (1996).

154. Dybtsev, D. N., Chun, H. \& Kim, K. Three-dimensional metal-organic framework with $(3,4)$-connected net, synthesized from an ionic liquid medium. Chem. Commun., 1594-1595 (2004).

155. Chen, B. et al. $\mathrm{Cu}_{2}(\mathrm{ATC}) \cdot 6 \mathrm{H}_{2} \mathrm{O}$ : Design of Open Metal Sites in Porous Metal-Organic Crystals (ATC: 1,3,5,7Adamantane Tetracarboxylate). J. Am. Chem. Soc. 122, 11559-11560 (2000).

156. Zheng, M., Liu, Y., Wang, C., Liu, S. \& Lin, W. Cavity-induced enantioselectivity reversal in a chiral metalorganic framework Brønsted acid catalyst. Chem. Sci. 3, 2623-2627 (2012).

157. Ma, S., Wang, X.-S., Collier, C. D., Manis, E. S. \& Zhou, H.-C. Ultramicroporous Metal-Organic Framework Based on 9,10-Anthracenedicarboxylate for Selective Gas Adsorption. Inorg. Chem. 46, 8499-8501 (2007).

158. Sudik, A. C., Côté, A. P., Wong-Foy, A. G., O'Keeffe, M. \& Yaghi, O. M. A Metal-Organic Framework with a Hierarchical System of Pores and Tetrahedral Building Blocks. Angew. Chem. Int. Ed. 45, 2528-2533 (2006).

159. Chen, Z. et al. Reticular Access to Highly Porous acs-MOFs with Rigid Trigonal Prismatic Linkers for Water Sorption. J. Am. Chem. Soc. 141, 2900-2905 (2019).

160. Chen, Z. et al. Balancing volumetric and gravimetric uptake in highly porous materials for clean energy. Science 368, 297-303 (2020).

161. Chun, H. \& Jung, H. Targeted Synthesis of a Prototype MOF Based on $\mathrm{Zn}_{4}(\mathrm{O})\left(\mathrm{O}_{2} \mathrm{C}\right)_{6}$ Units and a Nonlinear Dicarboxylate Ligand. Inorg. Chem. 48, 417-419 (2009).

162. Chae, H. K., Kim, J., Friedrichs, O. D., O’Keeffe, M. \& Yaghi, O. M. Design of Frameworks with Mixed Triangular and Octahedral Building Blocks Exemplified by the Structure of $\left[\mathrm{Zn}_{4} \mathrm{O}(\mathrm{TCA})_{2}\right]$ Having the Pyrite Topology. Angew. Chem. Int. Ed. 42, 3907-3909 (2003).

163. Park, K. S. et al. Exceptional chemical and thermal stability of zeolitic imidazolate frameworks. Proc. Natl Acad. Sci. USA 103, 10186-10191 (2006).

164. Schulz, M. et al. A Calixarene-Based Metal-Organic Framework for Highly Selective $\mathrm{NO}_{2}$ Detection. Angew. Chem. Int. Ed. 57, 12961-12965 (2018).

165. Han, Y. et al. In-Situ Ligand Formation-Driven Preparation of a Heterometallic Metal-Organic Framework for Highly Selective Separation of Light Hydrocarbons and Efficient Mercury Adsorption. ACS Applied Materials \& Interfaces 8, 23331-23337 (2016). 
166. Sun, D., Collins, D. J., Ke, Y., Zuo, J.-L. \& Zhou, H.-C. Construction of Open Metal-Organic Frameworks Based on Predesigned Carboxylate Isomers: From Achiral to Chiral Nets. Chemistry - A European Journal 12, 3768-3776 (2006).

167. Yuan, S. et al. Sequential Linker Installation: Precise Placement of Functional Groups in Multivariate Metal-Organic Frameworks. J. Am. Chem. Soc. 137, 3177-3180 (2015).

168. Deria, P. et al. Framework-Topology-Dependent Catalytic Activity of Zirconium-Based (Porphinato)zinc(II) MOFs. J. Am. Chem. Soc. 138, 14449-14457 (2016).

169. Feng, D. et al. Zirconium-Metalloporphyrin PCN-222: Mesoporous Metal-Organic Frameworks with Ultrahigh Stability as Biomimetic Catalysts. Angew. Chem. Int. Ed. 51, 10307-10310 (2012).

170. Li, P. et al. Hierarchically Engineered Mesoporous Metal-Organic Frameworks toward Cell-free Immobilized Enzyme Systems. Chem 4, 1022-1034 (2018).

171. Thiam, Z. et al. Extension of the Surface Organometallic Chemistry to Metal - Organic Framework: development of well-defined single site $\left[(\equiv \mathrm{Zr}-\mathrm{O}-) \mathrm{W}(=\mathrm{O})\left(\mathrm{CH}_{2}{ }^{t} \mathrm{Bu}\right)_{3}\right]$ olefin metathesis catalyst. J. Am. Chem. Soc. 142, 16690-16703 (2020).

172. Jiang, H.-L. et al. An Exceptionally Stable, Porphyrinic Zr Metal-Organic Framework Exhibiting pHDependent Fluorescence. J. Am. Chem. Soc. 135, 13934-13938 (2013).

173. Zhang, Y.-Z. et al. Nanocage containing metal-organic framework constructed from a newly designed low symmetry tetra-pyrazole ligand. J. Coord. Chem. 69, 3242-3249 (2016).

174. Duan, X. et al. A microporous metal-organic framework of a rare sty topology for high $\mathrm{CH}_{4}$ storage at room temperature. Chem. Commun. 49, 2043-2045 (2013).

175. Lin, X. et al. High Capacity Hydrogen Adsorption in Cu(II) Tetracarboxylate Framework Materials: The Role of Pore Size, Ligand Functionalization, and Exposed Metal Sites. J. Am. Chem. Soc. 131, 2159-2171 (2009).

176. Wang, X.-S. et al. Three-Dimensional Porous Metal-Metalloporphyrin Framework Consisting of Nanoscopic Polyhedral Cages. J. Am. Chem. Soc. 133, 16322-16325 (2011).

177. Grünker, R. et al. Topological Diversity, Adsorption and Fluorescence Properties of MOFs Based on a Tetracarboxylate Ligand. Eur. J. Inorg. Chem. 2010, 3835-3841 (2010).

178. Mohideen, M. I. H. et al. Upgrading gasoline to high octane numbers using a zeolite-like metal-organic framework molecular sieve with ana-topology. Chem. Commun. 54, 9414-9417 (2018).

179. An, J. et al. Metal-adeninate vertices for the construction of an exceptionally porous metal-organic framework. Nature Communications 3, 604 (2012).

180. Li, T., Kozlowski, M. T., Doud, E. A., Blakely, M. N. \& Rosi, N. L. Stepwise Ligand Exchange for the Preparation of a Family of Mesoporous MOFs. J. Am. Chem. Soc. 135, 11688-11691 (2013).

181. Zhang, J., Chen, S., Zingiryan, A. \& Bu, X. Integrated Molecular Chirality, Absolute Helicity, and Intrinsic Chiral Topology in Three-Dimensional Open-Framework Materials. J. Am. Chem. Soc. 130, 17246-17247 (2008).

182. Zhang, J.-P., Horike, S. \& Kitagawa, S. A Flexible Porous Coordination Polymer Functionalized by Unsaturated Metal Clusters. Angew. Chem. Int. Ed. 46, 889-892 (2007).

183. Yuan, S. et al. Retrosynthesis of multi-component metal-organic frameworks. Nature Communications $\mathbf{9}$, 808 (2018).

184. Furukawa, H. et al. Ultrahigh Porosity in Metal-Organic Frameworks. Science 329, 424-428 (2010).

185. Liu, L., Konstas, K., Hill, M. R. \& Telfer, S. G. Programmed Pore Architectures in Modular Quaternary Metal-Organic Frameworks. J. Am. Chem. Soc. 135, 17731-17734 (2013).

186. Liu, L. \& Telfer, S. G. Systematic Ligand Modulation Enhances the Moisture Stability and Gas Sorption Characteristics of Quaternary Metal-Organic Frameworks. J. Am. Chem. Soc. 137, 3901-3909 (2015).

187. Jiang, J., Furukawa, H., Zhang, Y.-B. \& Yaghi, O. M. High Methane Storage Working Capacity in MetalOrganic Frameworks with Acrylate Links. J. Am. Chem. Soc. 138, 10244-10251 (2016).

188. Liang, C.-C. et al. Engineering of Pore Geometry for Ultrahigh Capacity Methane Storage in Mesoporous Metal-Organic Frameworks. J. Am. Chem. Soc. 139, 13300-13303 (2017).

189. Hönicke, I. M. et al. Balancing Mechanical Stability and Ultrahigh Porosity in Crystalline Framework Materials. Angew. Chem. Int. Ed. 57, 13780-13783 (2018).

190. Han, D. et al. A non-interpenetrated porous metal-organic framework with high gas-uptake capacity. Chem. Commun. 47, 9861-9863 (2011).

191. Klein, N. et al. Route to a Family of Robust, Non-interpenetrated Metal-Organic Frameworks with ptolike Topology. Chemistry - A European Journal 17, 13007-13016 (2011).

192. Koh, K., Wong-Foy, A. G. \& Matzger, A. J. A Crystalline Mesoporous Coordination Copolymer with High Microporosity. Angew. Chem. Int. Ed. 47, 677-680 (2008). 
193. Muldoon, P. F. et al. Programmable Topology in New Families of Heterobimetallic Metal-Organic Frameworks. J. Am. Chem. Soc. 140, 6194-6198 (2018).

194. Pang, Q., Tu, B. \& Li, Q. Metal-organic frameworks with multicomponents in order. Coord. Chem. Rev. 388, 107-125 (2019).

195. Furukawa, H., Müller, U. \& Yaghi, O. M. "Heterogeneity within Order" in Metal-Organic Frameworks. Angew. Chem. Int. Ed. 54, 3417-3430 (2015).

196. Xu, W. et al. Anisotropic reticular chemistry. Nature Reviews Materials, 5, 764-779 (2020).

197. Zhai, Q.-G., Bu, X., Zhao, X., Li, D.-S. \& Feng, P. Pore Space Partition in Metal-Organic Frameworks. Acc. Chem. Res. 50, 407-417 (2017).

198. Zhao, X. et al. Multivariable Modular Design of Pore Space Partition. J. Am. Chem. Soc. 138, 15102-15105 (2016).

199. Chae, H. K. et al. A route to high surface area, porosity and inclusion of large molecules in crystals. Nature 427, 523-527 (2004).

200. Zhang, Y.-B. et al. Introduction of Functionality, Selection of Topology, and Enhancement of Gas Adsorption in Multivariate Metal-Organic Framework-177. J. Am. Chem. Soc. 137, 2641-2650 (2015).

201. Férey, G. et al. A Hybrid Solid with Giant Pores Prepared by a Combination of Targeted Chemistry, Simulation, and Powder Diffraction. Angew. Chem. Int. Ed. 43, 6296-6301 (2004).

202. Park, Y. K. et al. Crystal Structure and Guest Uptake of a Mesoporous Metal-Organic Framework Containing Cages of 3.9 and 4.7 nm in Diameter. Angew. Chem. Int. Ed. 46, 8230-8233 (2007).

203. Fang, Q. et al. A Metal-Organic Framework with the Zeolite MTN Topology Containing Large Cages of Volume $2.5 \mathrm{~nm}^{3}$. Angew. Chem. Int. Ed. 44, 3845-3848 (2005).

204. Mallick, A. et al. Unprecedented Ultralow Detection Limit of Amines using a Thiadiazole-Functionalized Zr(IV)-Based Metal-Organic Framework. J. Am. Chem. Soc. 141, 7245-7249 (2019).

205. Cohen, S. M. The Postsynthetic Renaissance in Porous Solids. J. Am. Chem. Soc. 139, 2855-2863 (2017).

206. Xue, D.-X. et al. Tunable Rare Earth fcu-MOF Platform: access to Adsorption Kinetics Driven Gas/Vapor Separations via Pore Size Contraction. J. Am. Chem. Soc. 137, 5034-5040 (2015).

207. Deng, H. et al. Multiple Functional Groups of Varying Ratios in Metal-Organic Frameworks. Science 327, 846-850 (2010).

208. Kong, X. et al. Mapping of Functional Groups in Metal-Organic Frameworks. Science 341, 882-885 (2013).

209. Fracaroli, A. M. et al. Seven Post-synthetic Covalent Reactions in Tandem Leading to Enzyme-like Complexity within Metal-Organic Framework Crystals. J. Am. Chem. Soc. 138, 8352-8355 (2016).

210. Feng, L. et al. Creating Hierarchical Pores by Controlled Linker Thermolysis in Multivariate Metal-Organic Frameworks. J. Am. Chem. Soc. 140, 2363-2372 (2018).

211. Wang, L. J. et al. Synthesis and Characterization of Metal-Organic Framework-74 Containing 2, 4, 6, 8, and 10 Different Metals. Inorg. Chem. 53, 5881-5883 (2014).

212. Luo, T.-Y. et al. Multivariate Stratified Metal-Organic Frameworks: Diversification Using Domain Building Blocks. J. Am. Chem. Soc. 141, 2161-2168 (2019).

213. Feng, L. et al. Imprinted Apportionment of Functional Groups in Multivariate Metal-Organic Frameworks. J. Am. Chem. Soc. 141, 14524-14529 (2019).

214. Feng, L. et al. Lattice Expansion and Contraction in Metal-Organic Frameworks by Sequential Linker Reinstallation. Matter 1, 156-167 (2019).

215. Lin, G. et al. 3D Porphyrin-Based Covalent Organic Frameworks. J. Am. Chem. Soc. 139, 8705-8709 (2017).

216. Ma, T. et al. Single-crystal x-ray diffraction structures of covalent organic frameworks. Science 361, 4852 (2018).

\section{Acknowledgements}

The authors gratefully acknowledge financial support from the King Abdullah University of Science and Technology (KAUST) and thank V. Guillerm for helpful comments and suggestions.

\section{Author contributions}

H.J. and M.E. formulated the content of this Review. All authors contributed to the writing and editing of the manuscript prior to submission. 


\section{Competing interests}

The authors declare no competing interests.

Peer review information

Nature Reviews Materials thanks the anonymous reviewers for their contribution to the peer review of this work. 
Table 1 | Summary of three-periodic and two-periodic edge-transitive nets

\begin{tabular}{|c|c|c|}
\hline $\begin{array}{l}\text { Highest } \\
\text { coordination } \\
\text { number }\end{array}$ & Coordination & $\begin{array}{l}\text { Edge-transitive nets } \\
\text { by coordination figure }{ }^{\text {a }}\end{array}$ \\
\hline 3 & $3-c$ & $3: \mathbf{s r s}, \mathbf{h c b}^{\mathrm{b}}$ \\
\hline 4 & $\begin{array}{l}4-c \\
(3,4)-c \\
(4,4)-c\end{array}$ & $\begin{array}{l}\text { S: nbo, lvt, rhr, sql }{ }^{\mathrm{b}}, \mathbf{k g m}^{\mathrm{b}} \text {; T: dia, lcs, lcv, qtz, sod, ana } \\
\text { 3S: tbo, pto; 3T: bor, ctn } \\
\text { SS: ssa, ssb; ST: pth, pts; TT: ssc }\end{array}$ \\
\hline & $6-c$ & $\mathrm{H}: \mathbf{h x g}, \mathbf{h x} \mathbf{l}^{\mathrm{b}} ; \mathrm{O}: \mathbf{p c u}, \mathbf{b c s}, \mathbf{c r s} ; \mathrm{P}: \mathbf{a c s}, \mathbf{l c y}$ \\
\hline 6 & $\begin{array}{l}(3,6)-c \\
(4,6)-c \\
(6,6)-c\end{array}$ & $\begin{array}{l}\text { 3O: pyr, spn; } 3 \mathrm{H}: \mathbf{k g d}^{\mathrm{b}} \\
\text { SH: she; SO: soc; SP: stp; TO: gar, toc, iac; TP: ifi } \\
\text { OP: nia }\end{array}$ \\
\hline 8 & $\begin{array}{l}8-c \\
(3,8)-c \\
(4,8)-c \\
(6,8)-c\end{array}$ & $\begin{array}{l}\text { C: bcu, reo; A: thp } \\
\text { 3C: the } \\
\text { SC: scu, csq, sqc; TC: flu } \\
\text { OC: ocu }\end{array}$ \\
\hline 12 & $\begin{array}{l}12-\mathrm{c} \\
(3,12)-\mathrm{c} \\
(4,12)-\mathrm{c} \\
(6,12)-\mathrm{c}\end{array}$ & $\begin{array}{l}\text { B: fcu } \\
\text { 3U: ttt } \\
\text { SB: ftw; SX: shp; TI: ith } \\
\text { HU: mgc; PX: alb }\end{array}$ \\
\hline 24 & $\begin{array}{l}(3,24)-c \\
(4,24)-c\end{array}$ & $\begin{array}{l}\text { 3R: rht } \\
\text { TK: twf }\end{array}$ \\
\hline
\end{tabular}

Only basic edge-transitive nets are listed in this Table. All 96 nets with one type of edge in the RCSR database can be found in Supplementary Information, including basic nets, binary nets, nets with intersecting edges, noncrystallographic (NC) nets and spn-z. ${ }^{a}$ Coordination figures: $3=$ triangle, $\mathrm{S}=$ square, $\mathrm{T}=$ tetrahedron, $\mathrm{H}=$ hexagon, $\mathrm{O}=$ octahedron, $\mathrm{P}=$ trigonal prism, $\mathrm{C}=$ cube, $\mathrm{A}=$ square antiprism or bisdisphenoid, $\mathrm{B}=$ cuboctahedron, $\mathrm{U}=$ truncated tetrahedron, $\mathrm{X}=$ hexagonal prism, $\mathrm{I}=$ icosahedron, $\mathrm{R}=$ rhombicuboctahedron, $\mathrm{K}=$ truncated octahedron. ${ }^{\mathrm{b}}$ Two-periodic edge-transitive net. 
Building units Building blocks

a

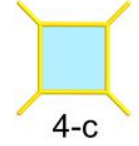

square

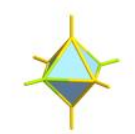

6-c octahedron

b

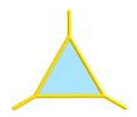

3-c

triangle

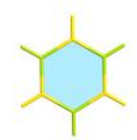

6-c

hexagon

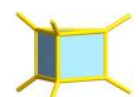

6-c

trigonal prism

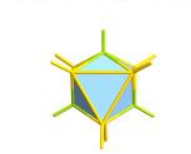

9-c

tricapped trigonal prism

$\left(\mathrm{J}_{51}\right)$

j

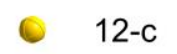

(- $6-\mathrm{c}$

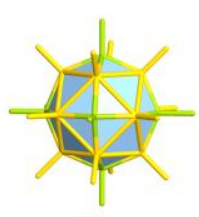

18-c
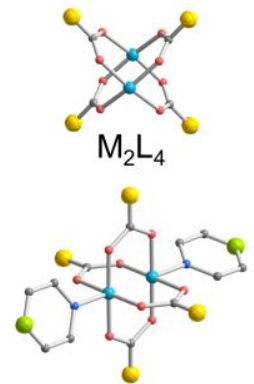

$\mathrm{M}_{2} \mathrm{~L}_{4} \mathrm{~L}_{2}^{\prime}$
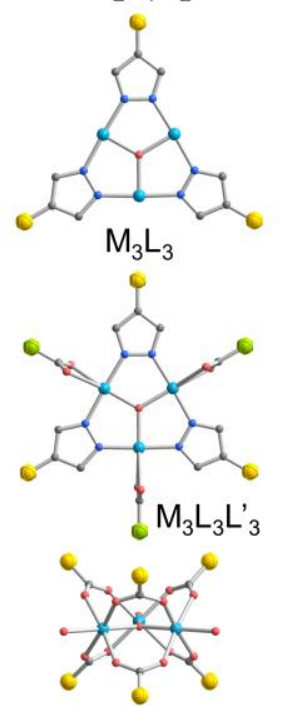

$\mathrm{M}_{3} \mathrm{~L}_{6}$ d

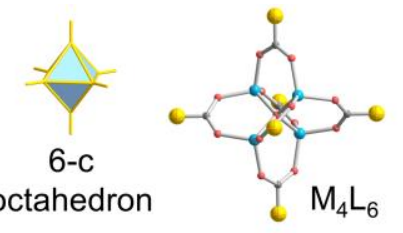

e<smiles>C=C1CC2=CC1C2</smiles>

8-c

cuboid

f

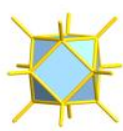

12-c cuboctahedro
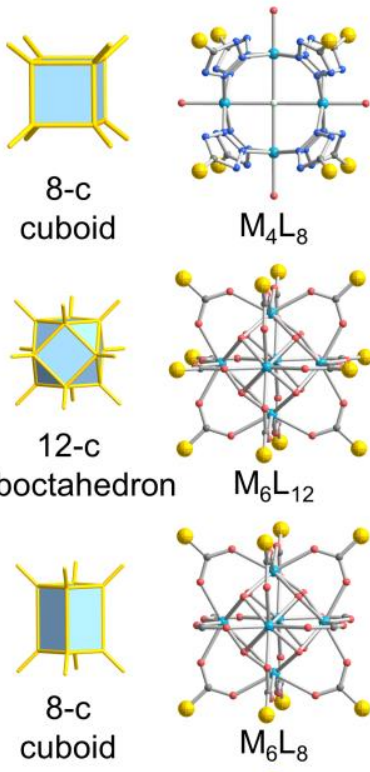

$\mathrm{M}_{4} \mathrm{~L}_{8}$

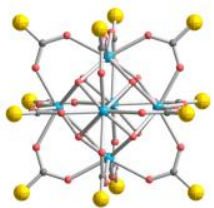

n $\quad M_{6} L_{12}$
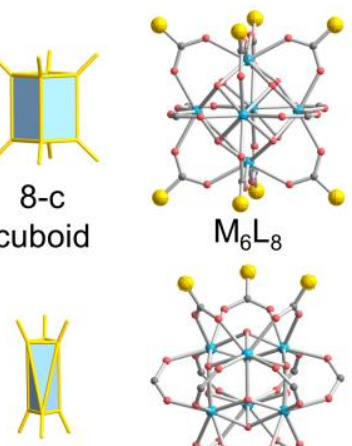

6-c

trigonal antiprism $\quad M_{6} \mathrm{~L}_{6}$
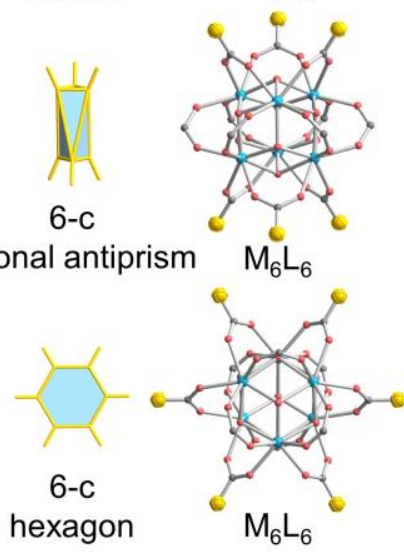

k g

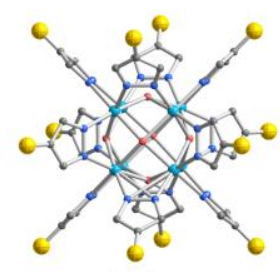

12-c

cuboctahedron

h

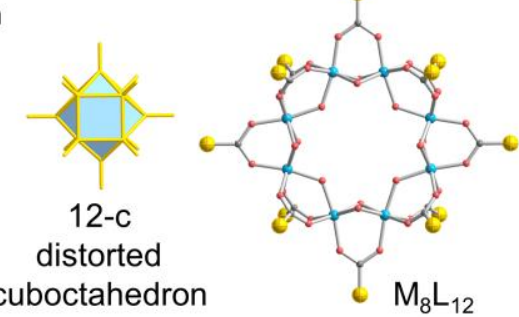

cuboctahedron

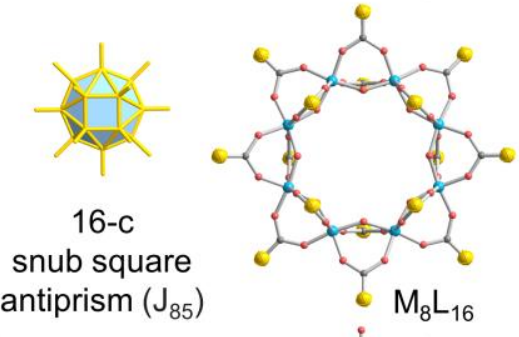

i
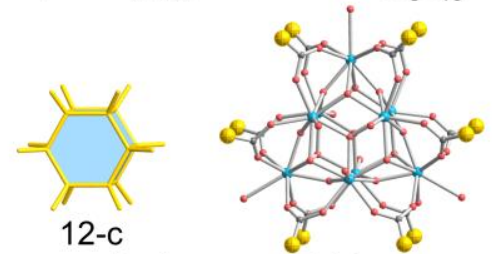

hexagonal prism

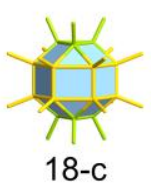

elongated trigonal

orthobicupola $\left(\mathrm{J}_{35}\right)$
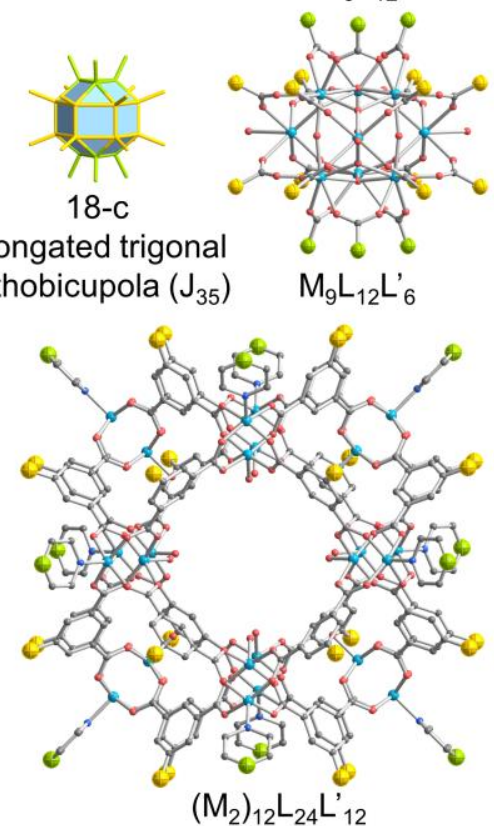

Fig. 1| Representative building blocks that can be reliably obtained. Illustration of building units obtained from the same types of building blocks. Colour scheme: metals (light blue), carbon (grey), oxygen (red), nitrogen (dark blue), halogens (cyan), basic points of expansion (yellow), extra points of expansion (green). L, linker; M, metal; $n$-c, $n$-connected $(n=3-36)$. 


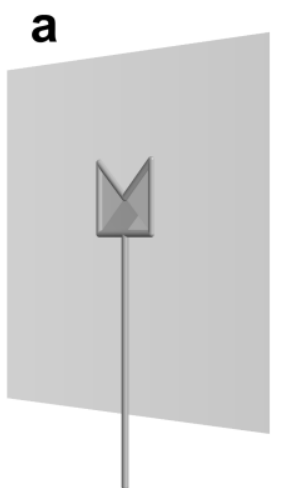

identity

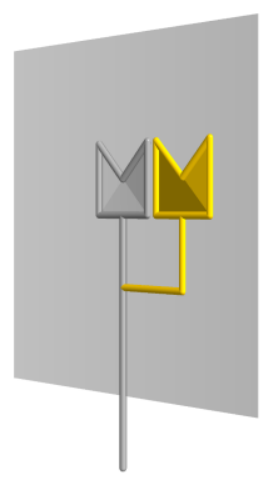

in-plane translation

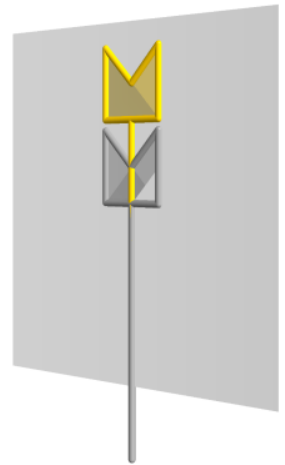

expansion

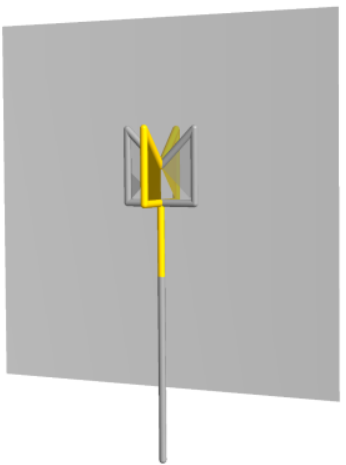

torsion

b

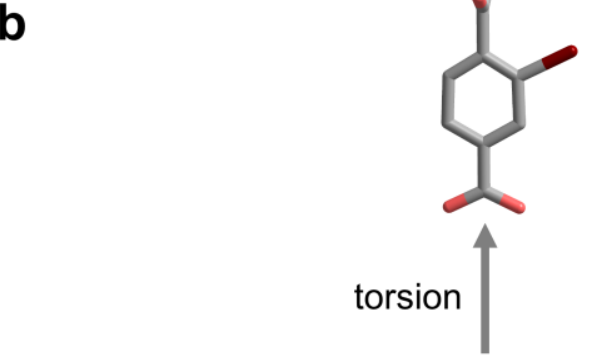

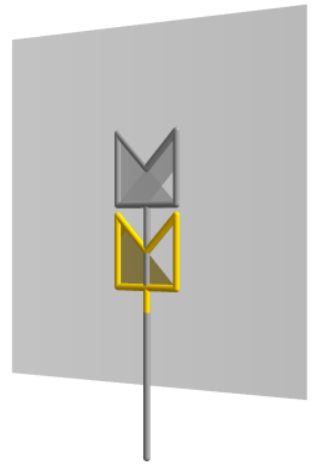

contraction

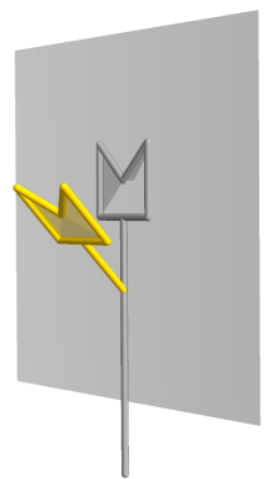

out-of-plane rotation

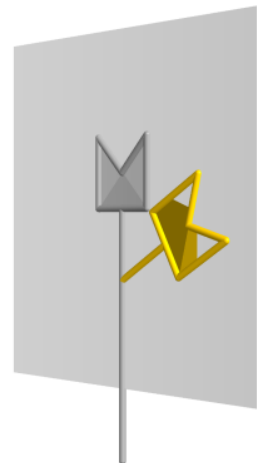

in-plane rotation

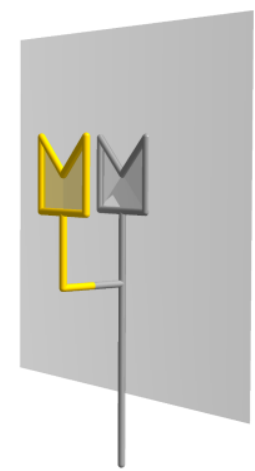

out-of-plane translation<smiles>[X][Ca]</smiles>

identity<smiles>[Tl]</smiles>

out-of-plane translation

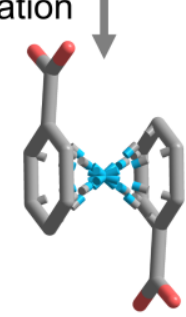

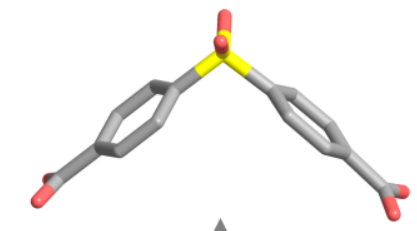

$$
\begin{gathered}
\text { out-of-plane } \\
\text { rotation }
\end{gathered}
$$
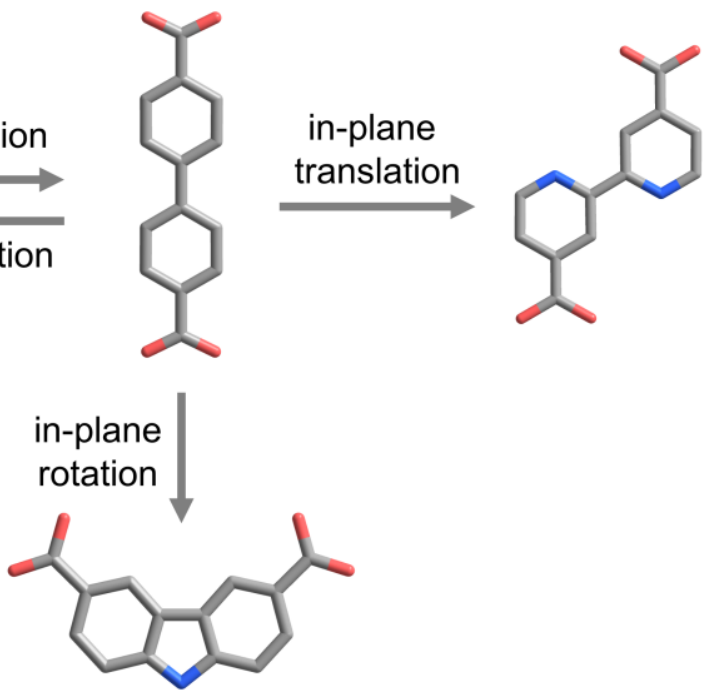

Fig. 2 Transformations of the position of linking groups in organic linkers. a | The eight fundamental operations, where grey and yellow indicate the linking group before and after the operation, respectively. $\mathbf{b}$ | Examples of ditopic linkers, illustrating the different transformations. Colour scheme: carbon (grey), oxygen (red), nitrogen (blue), sulfur (yellow). For clarity, the hydrogen atoms are omitted. 


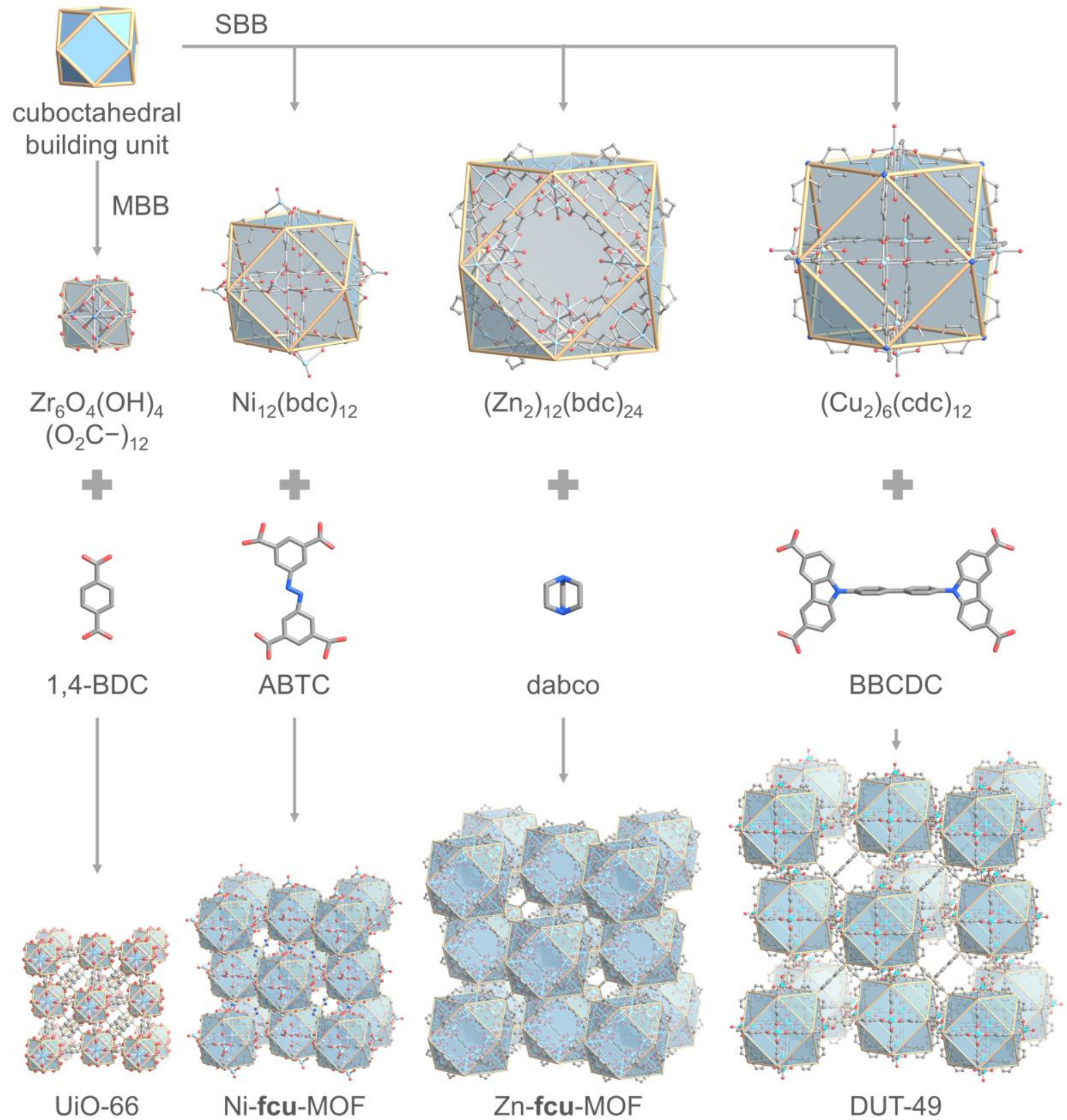

Fig. 3 The formation of fcu-MOFs through the MBB and the SBB approaches. Schematic showing the molecular building block (MBB) approach for the assembly of UiO-66 and the supermolecular building block (SBB) approach for the assembly of Ni-fcu-MOF, Zn-fcu-MOF and DUT-49. Hydrogen atoms and nontopological functional groups are omitted for clarity. ABTC, 3,3',5,5'-azobenzene tetracarboxylate; BBCDC, 9, $9^{\prime}$ ([1,1'-biphenyl]-4,4'-diyl)bis(9H-carbazole-3,6-dicarboxylate; BDC, benzene dicarboxylate; CDC, 9Hcarbazole-3,6-dicarboxylate; dabco, 1,4-diazabicyclo-[2.2.2]octane. 
a

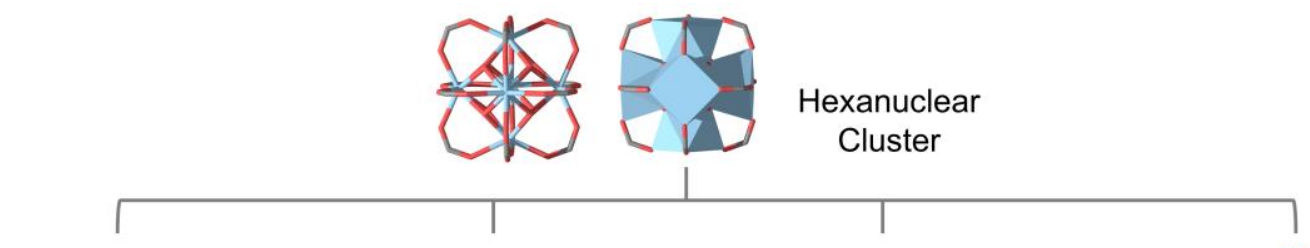

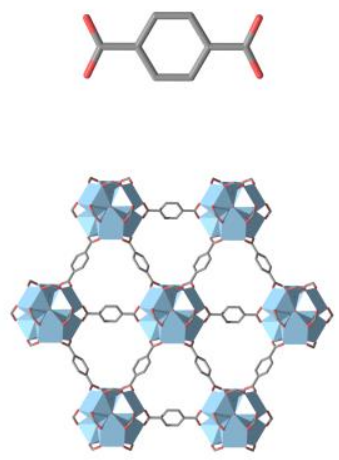

hxI-SBL<smiles>CC(C)C1CCCC(C(C)C)C1</smiles>

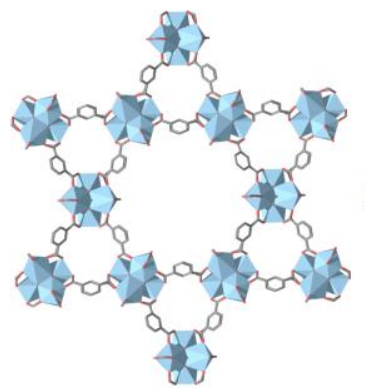

kgm-SBL
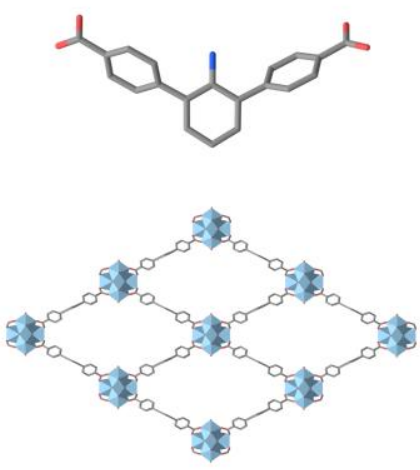

sql-SBL
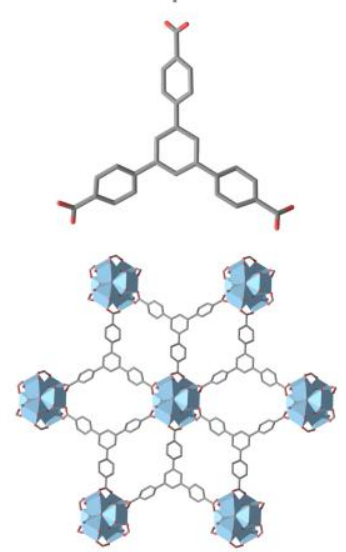

kgd-SBL

b

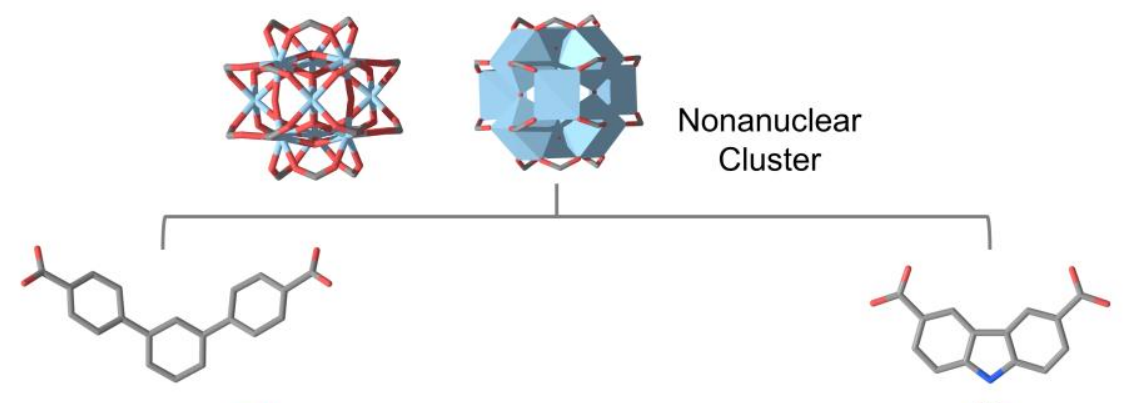

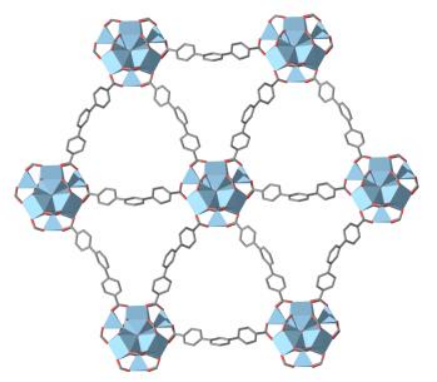

hxl-SBL

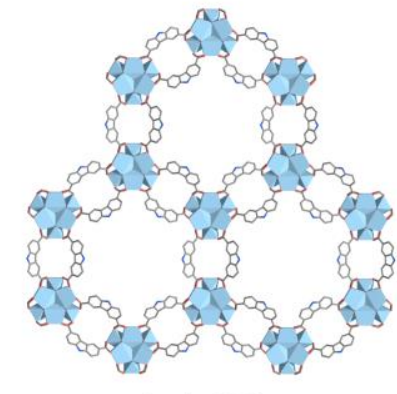

hcb-SBL

Fig. 4 | Supermolecular building layers based on hexanuclear and nonanuclear clusters. a | Supramolecular building layers (SBLs) based on a hexanuclear cluster. b $\mid$ SBLs based on a nonanuclear cluster. 


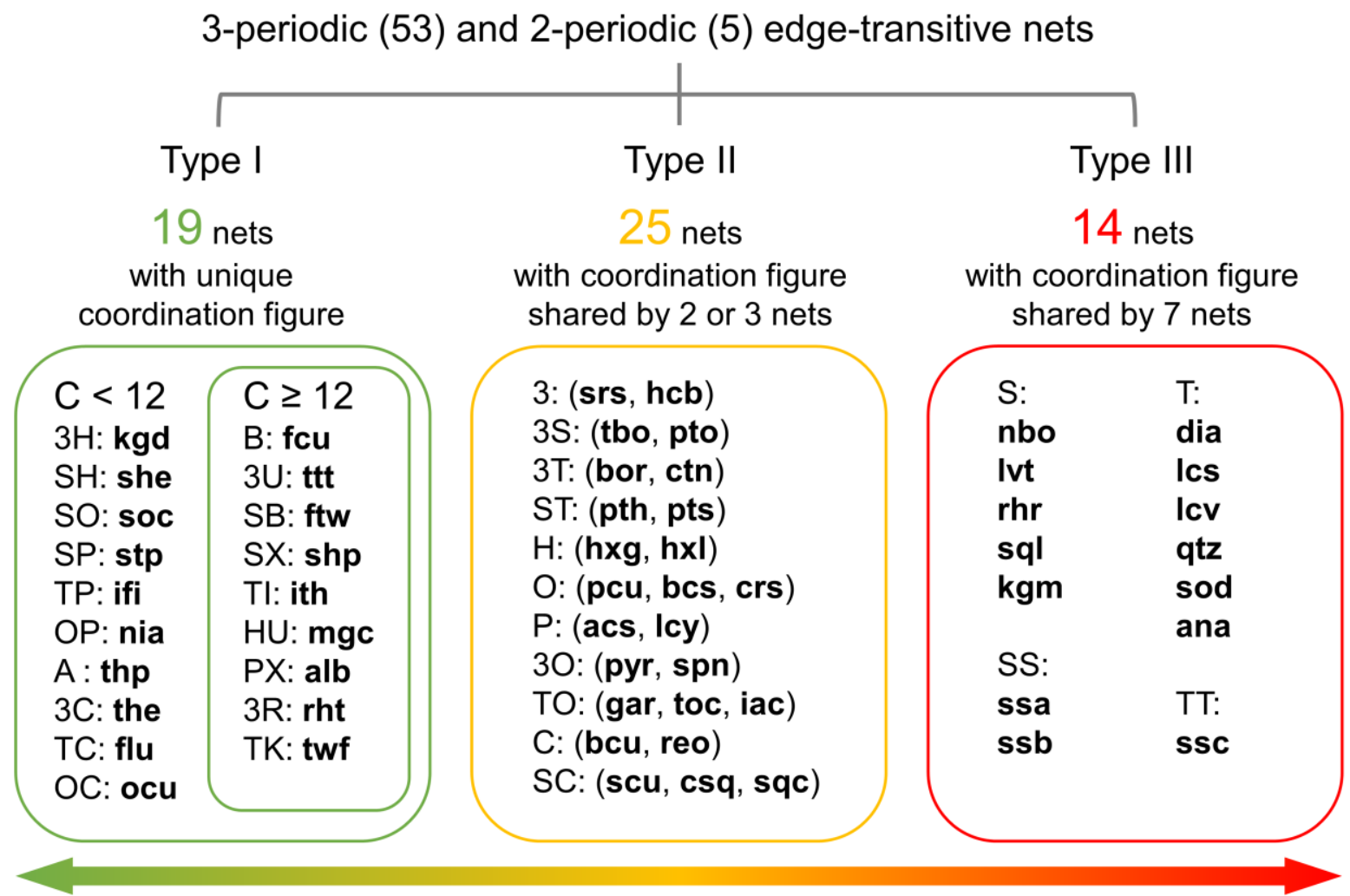

Expressed net-coded building units (net-cBU)

Dormant net-coded building units (net-cBU)

Fig. 5 | Categories of edge-transitive nets. Edge-transitive three-periodic nets (53 nets) and two-periodic nets (five nets) are categorized into three types by considering the uniqueness of the building unit combinations. Type I nets have a unique coordination figure (that is, shape of the building unit combinations). All highly coordinated nets (highest coordination number $C \geq 12$ ) are reticulated by unique building unit combinations. Type II nets have a coordination figure coded by two or three nets. Type III nets comprise only square or tetrahedral building units, each of which is coded by seven nets. Coordination figures: $3=$ triangle, $\mathrm{S}=$ square, $\mathrm{T}=$ tetrahedron, $\mathrm{H}=$ hexagon, $\mathrm{O}=$ octahedron, $\mathrm{P}=$ trigonal prism, $\mathrm{C}=$ cube, $\mathrm{A}=$ square antiprism or bisdisphenoid, $\mathrm{B}=$ cuboctahedron, $\mathrm{U}=$ truncated tetrahedron, $\mathrm{X}=$ hexagonal prism, $\mathrm{I}=$ icosahedron, $\mathrm{R}=$ rhombicuboctahedron, $\mathrm{K}=$ truncated octahedron. 
a

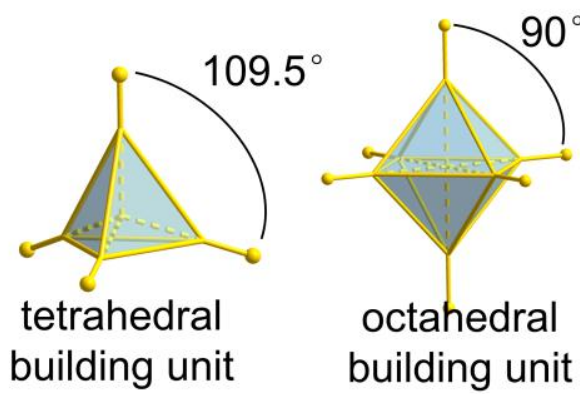

b

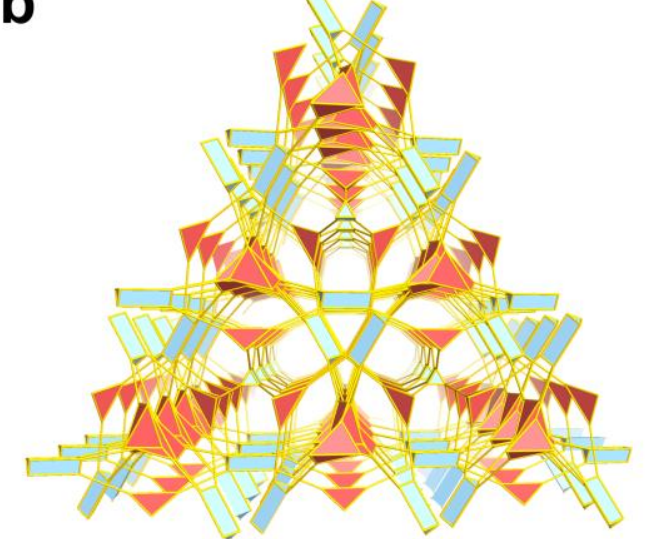

ifi-a net

C

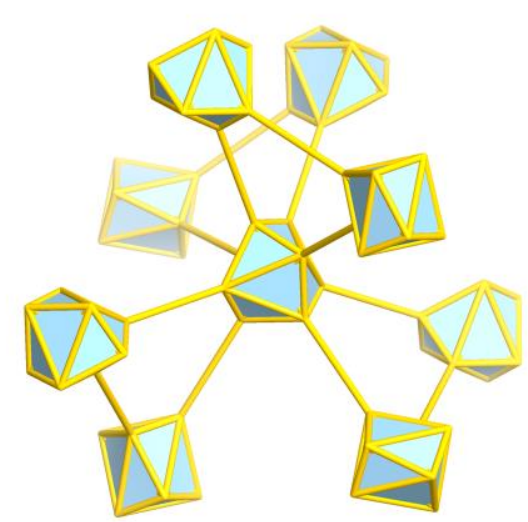

thp-a net

d

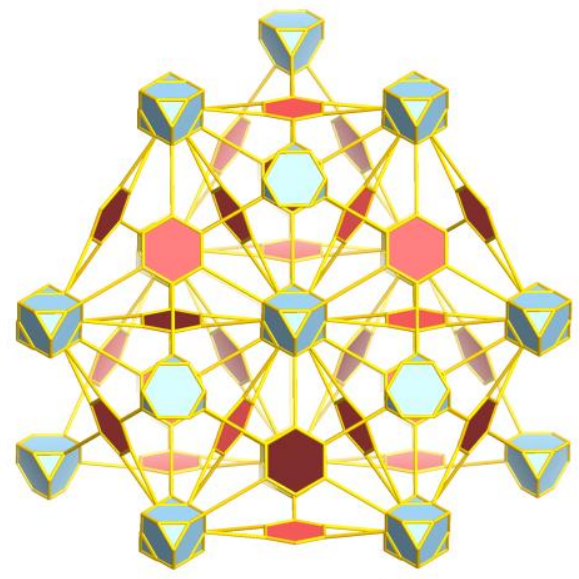

mgc-a net

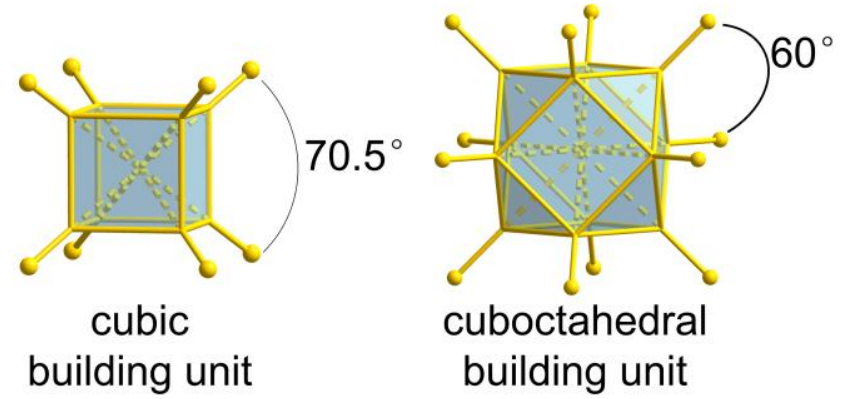

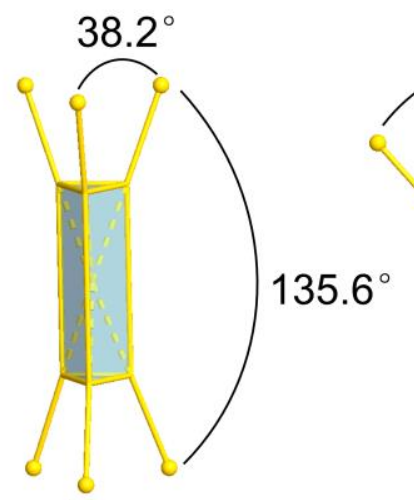

6-c

trigonal prismatic building unit

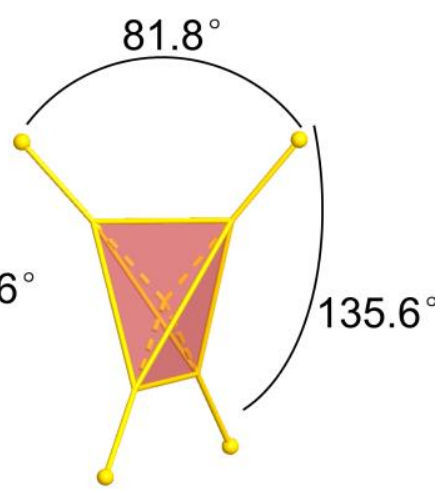

4-c tetrahedral building unit

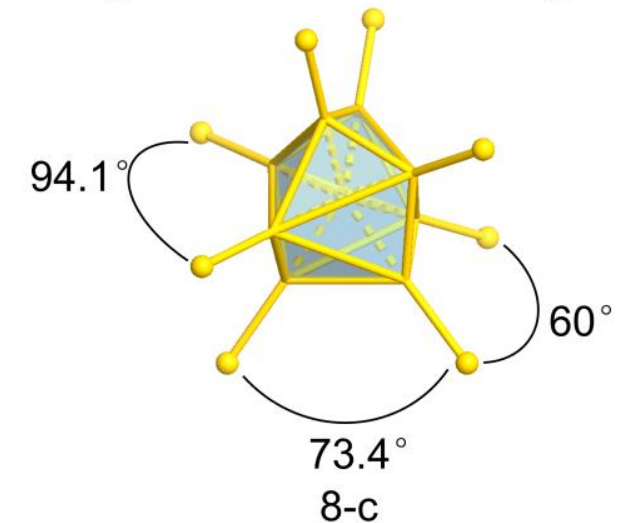

distorted bisdisphenoid building unit

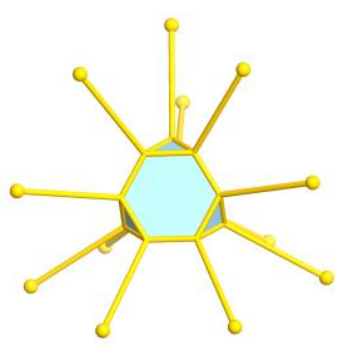

12-c

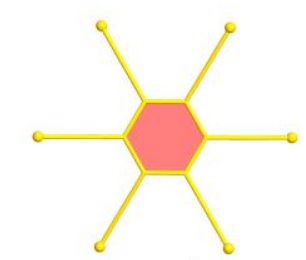

top view

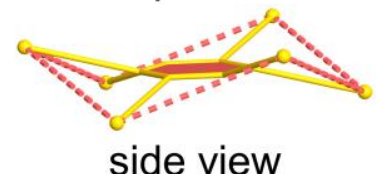

6-c

truncated tetrahedral distorted hexagonal building unit building unit 
Fig. 6 | Type I edge-transitive nets for the discovery of new metal-organic framework structures. a $\mid$ The vertex-centre-vertex angle in regular tetrahedral, octahedral, cubic and cuboctahedral building units. b | Structure of the ifi-a net and the corresponding trigonal prismatic and tetrahedral building units. $\mathbf{c} \mid$ Structure of the thp-a net and the distorted bisdisphenoid building unit. $\mathbf{d} \mid$ Structure of the mgc-a net and the truncated tetrahedral and distorted hexagonal building units. $n$-c, $n$-connected $(n=4-12)$. 

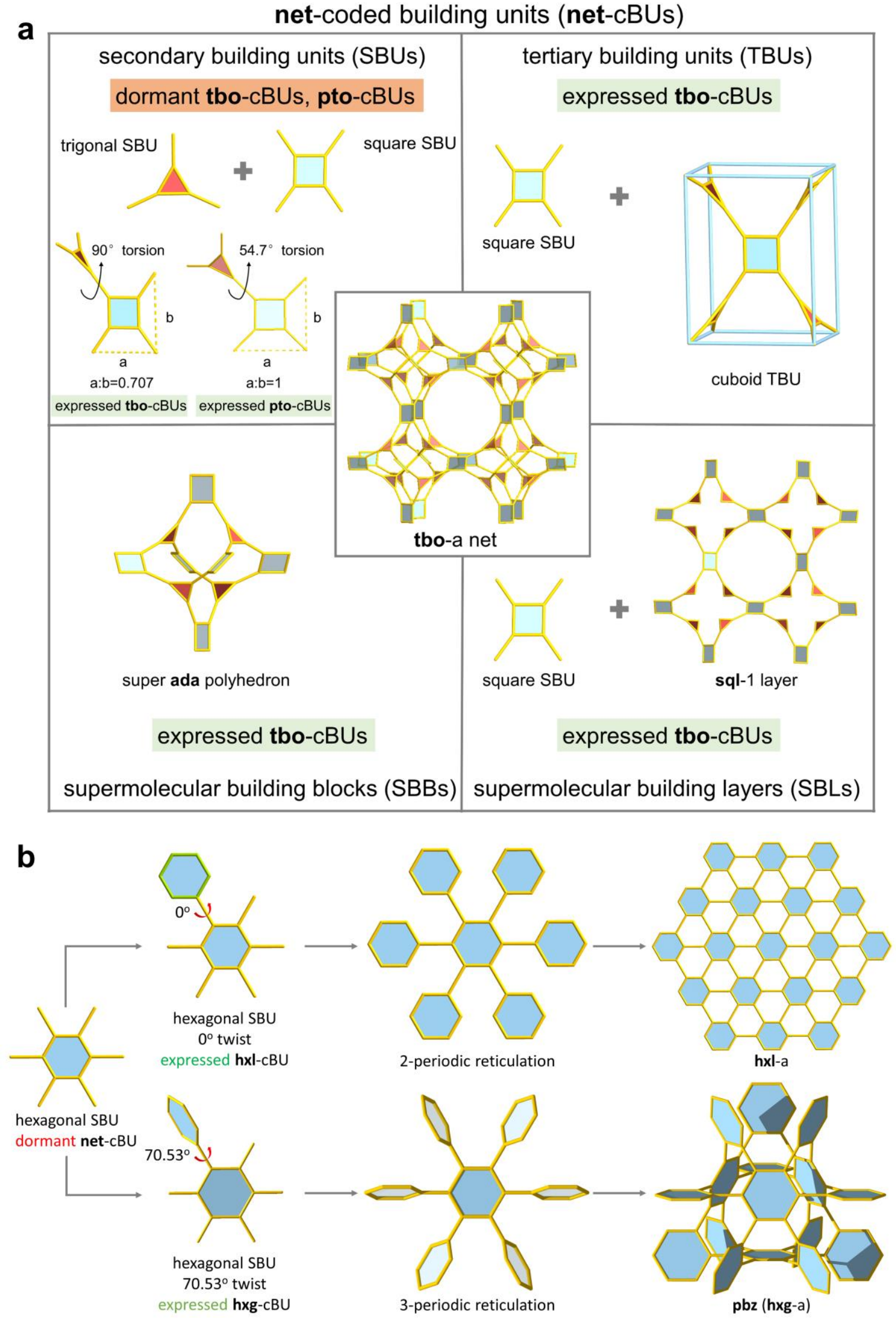

Fig. 7 | The concept of net-coded building units. a | The concept of net-coded building units (net-CBUs) demonstrated with the edge-transitive tbo net (shown as tbo-a net to highlight the geometry). In this case, there 
are four approaches for the design of tbo-MOFs, involving secondary building units (SBUs), tertiary building units (TBUs), supermolecular building blocks (SBBs) or supermolecular building layers (SBLs). b | Transformation of the hexagonal SBU, a dormant net-cBU, into an expressed hxl-cBU with a $0^{\circ}$ torsion angle or an expressed hxg-cBU with a $70.53^{\circ}$ torsion angle.

a

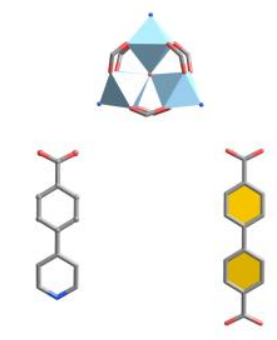

ncb-MOF-IIIc, ITC-4

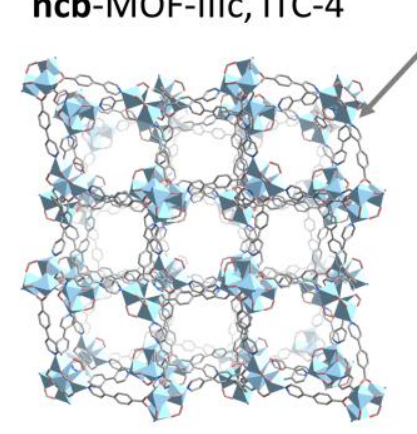

pcu part

3-periodic
$3 p-0 p$ merged

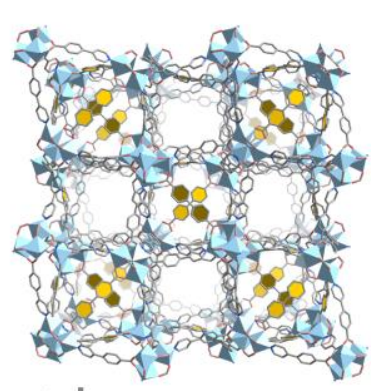

ncb

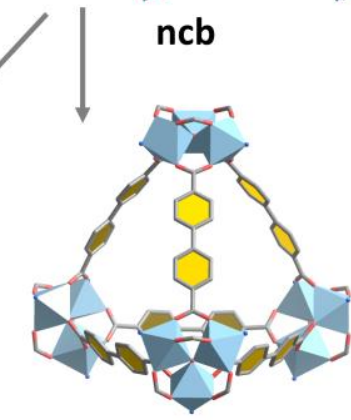

tet part

0-periodic
C

\section{$3 p-2 p$ merged}

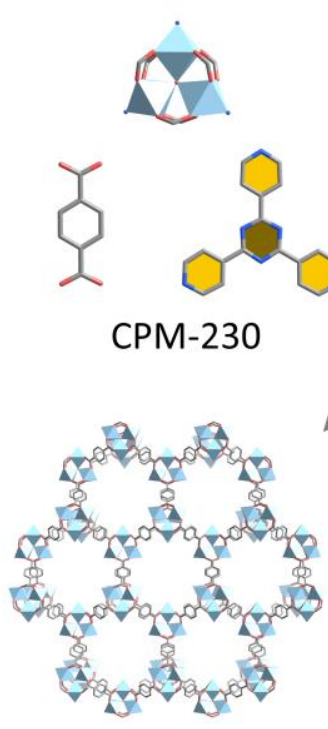

acs part

3-periodic
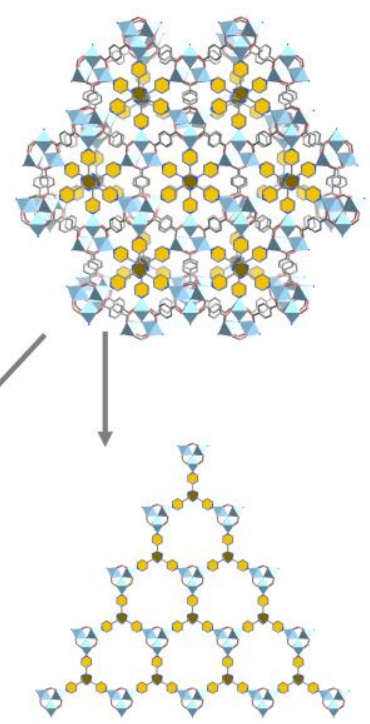

hcb part

2-periodic b

$3 p-1 p$ merged
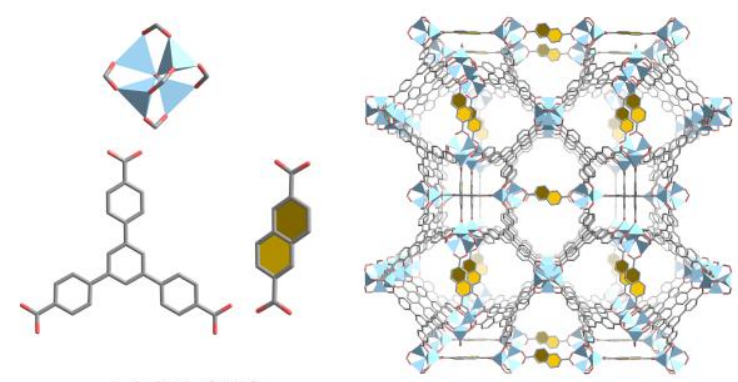

MOF-250

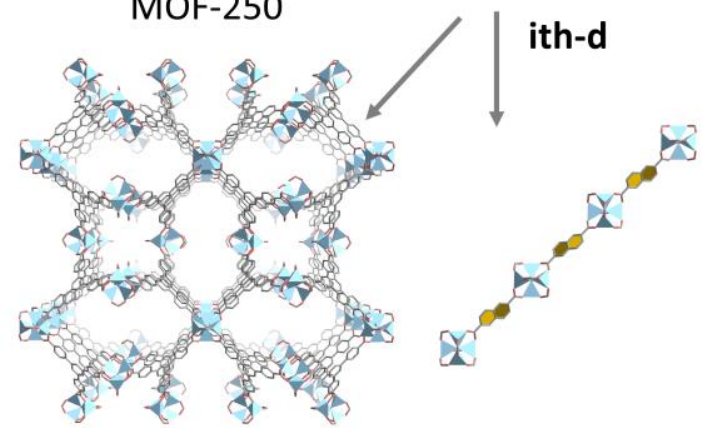

pto part

Icw part

3-periodic

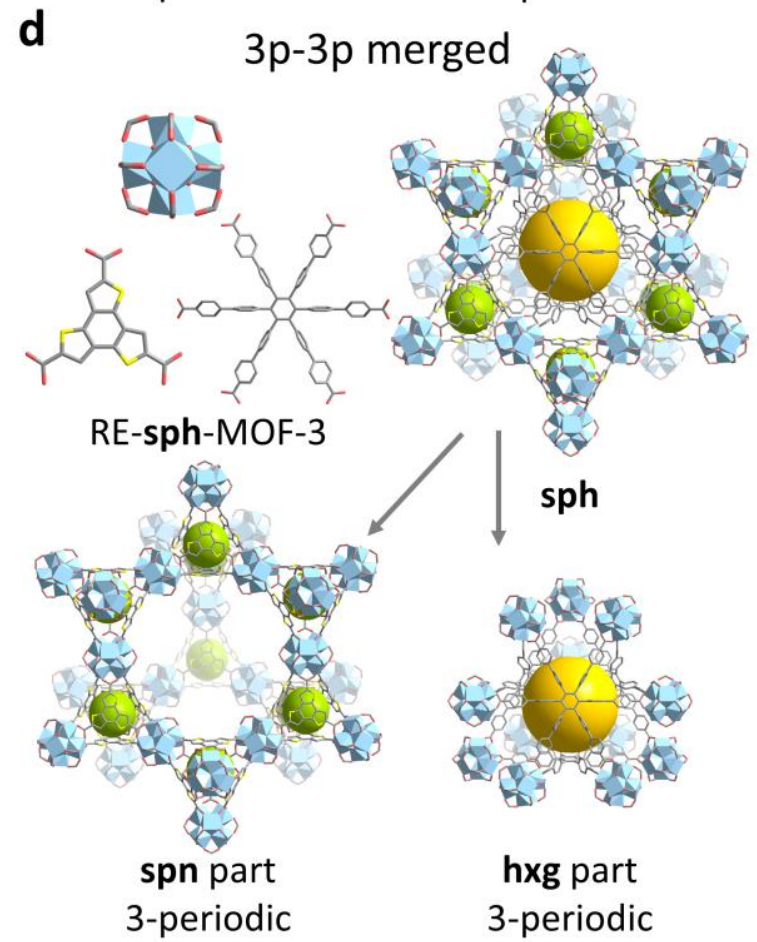

Fig. 8 | Example metal-organic frameworks based on merged nets. a | Structure of a 9-c ncb metal-organic framework (MOF) (IIIc, ITC-4), which can be split into two fragments: a three-periodic 6-c pcu framework and a zero-periodic 3-c tet framework. b | Structure of a (3,6)-c ith-d MOF (MOF-250), which can be split into a three-periodic (3,4)-c pto framework and a one-periodic 2-c lcw framework. $\mathbf{c} \mid$ Structure of a (3,9)-c MOF (CPM230), which can be split into a three-periodic 6-c acs framework and a two-periodic 3-c heb framework. d | 
Structure of a $(3,6,12)$-c sph MOF (RE-sph-MOF-3), which can be split into a three-periodic $(3,6)$-c spn framework and a three-periodic 6-c hxg framework. $n$-c, $n$-coordinated; RE, rare earth.

a

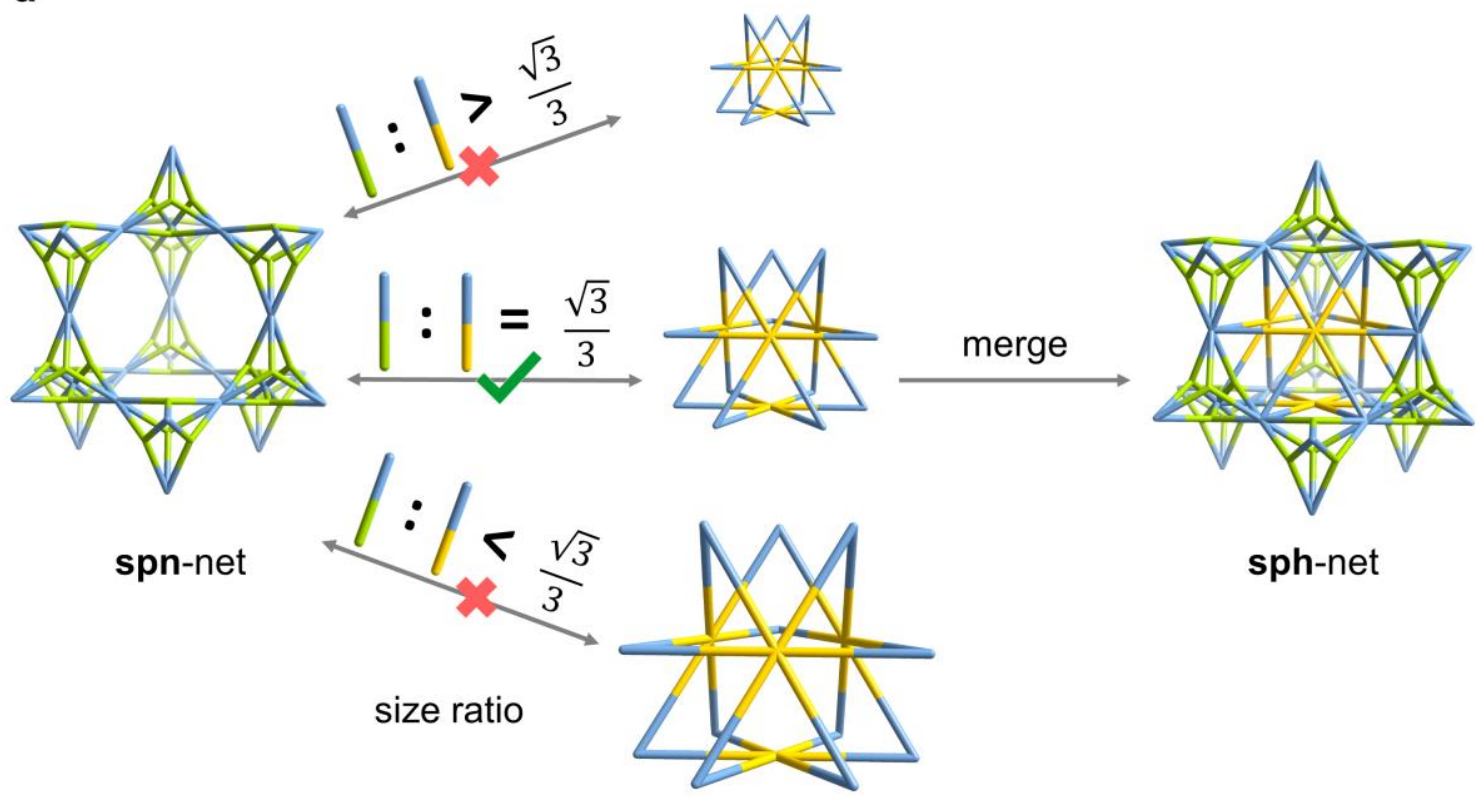

b

hxg-net

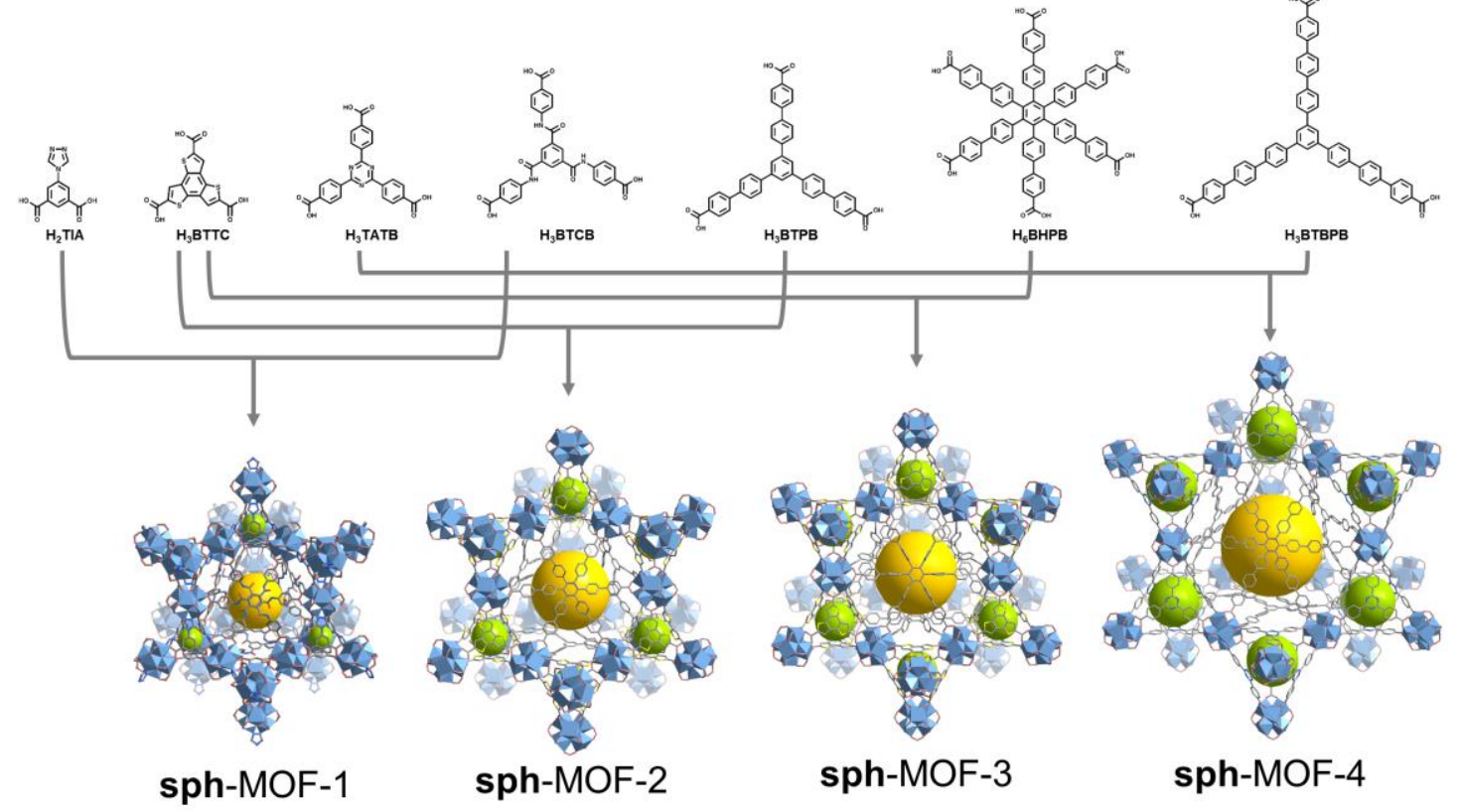

Fig. 9 Ratio constant for the isoreticular expansion of merged net structures. a $\mid$ Nets can only merge when the ratio of their edge lengths meets a constant, which is an intrinsic property of the merged net. In this example, when the edge ratio of the spn net and $\mathbf{h x g}$ net meets the ratio constant of $\sqrt{3} / 3$, they can merge to form the $\mathbf{s p h}$ net. $\mathbf{b}$ | The ratio constant can be used to predict the combination of linkers that can be used to form a series of mixed-linker RE-sph-MOFs ${ }^{10}$. $\mathrm{H}_{2}$ TIA, 5-(4H-1,2,4-triazol-4-yl)-isophthalic acid; $\mathrm{H}_{3} \mathrm{BTBPB}, 4,4^{\prime}, 4^{\prime \prime}$-(benzene1,3,5-triyl-tris(biphenyl-4,4'-diyl))tribenzoic $\quad$ acid; $\quad \mathrm{H}_{3} \mathrm{BTCB}, \quad 4,4^{\prime}, 4^{\prime \prime}$-((benzene-1,3,5tricarbonyl)tris(azanediyl))tribenzoic acid; $\mathrm{H}_{3}$ BTTC, benzo-tristhiophene carboxylic acid; $\mathrm{H}_{3} \mathrm{BTPB}, 4,4^{\prime}, 4^{\prime \prime}$ (benzene-1,3,5-triyl-tris(benzene-4,1-diyl))tribenzoic acid; $\mathrm{H}_{3} \mathrm{TATB}, 4,4^{\prime}, 4^{\prime \prime}$ - $s$-triazine-2,4,6-triyl-tribenzoic acid; $\mathrm{H}_{6} \mathrm{BHPB}$, hexakis(4-(4-carboxyphenyl)phenyl)benzene; RE, rare earth. 